\title{
Accrual mispricing, value-at-risk, and expected stock returns
}

\author{
Prodosh Simlai ${ }^{1}$
}

Accepted: 2 April 2021 / Published online: 26 April 2021

(c) The Author(s), under exclusive licence to Springer Science+Business Media, LLC, part of Springer Nature 2021

\begin{abstract}
We investigate the extent to which a parsimonious measure of maximum likely loss that captures the tail risk of returns_-known as value-at-risk (VaR) — explains the relationship between accruals and the cross-sectional dispersion of expected stock returns. We construct portfolios based on Sloan's (Account Rev 71(3):289-315, 1996) total accruals (TA) measure and individual asset-level VaR, which reflects the dynamic behavior of the asset distribution. We document that $\mathrm{VaR}$ is in congruence with portfolio-level accruals and that there is a significant positive relationship between VaR and the cross-section of portfolio returns. Allowing a double-sort involving VaR and TA further suggests that the spread between low- and high-TA portfolios is significantly attenuated after controlling for VaR. We also conduct a firm-level cross-sectional regression analysis and demonstrate that the TA- and VaR-based characteristics—but not the factor-mimicking portfolios-are compensated with higher expected returns, and that VaR neither subsumes nor is subsumed by TA. Finally, our cross-sectional decomposition analysis suggests that the firm-level VaR captures at least $7 \%$ of the accrual premium even in the presence of size and book-to-market. These findings lend support for the mispricing explanation of the accrual anomaly.
\end{abstract}

Keywords Accruals · Value-at-risk - Abnormal returns $\cdot$ Anomalies $\cdot$ Mispricing $\cdot$ Crosssection of returns

JEL Classification C4 $\cdot \mathrm{G} 11 \cdot \mathrm{G} 12 \cdot \mathrm{M} 41$

\section{Introduction}

Accruals are defined as the difference between accounting earnings and cash flows from operations. Since Graham and Dodd (1934), financial theory has emphasized that investors fixate too heavily on corporate earnings. Sloan (1996) has tested this investor fixation theory and documented a negative relationship between accounting accruals and stock returns (see e.g., Dechow et al. 2014 for a review). This relationship, known as the accrual anomaly or accrual effect, poses important challenges for rational asset pricing theories

Prodosh Simlai

prodosh.simlai@und.edu

1 Department of Economics and Finance, Nistler College of Business and Public Administration, University of North Dakota, 293 Centennial Drive, Grand Forks, ND 58202, USA 
(Hirshleifer et al. 2012), and has been investigated in a large number of studies (Ball et al. 2016; Stambaugh and Yuan 2017). One can classify various explanations of the accrual effect into three broad groups. ${ }^{1}$ The first group interprets the accrual anomaly as evidence of investors overestimating the persistence of the accrual component of earnings, while the second group argues in favor of a rational expectation approach for the interpretation of the accrual effect. The third group relates the accrual anomaly to investors' pricing of growth. Irrespective of the conclusions drawn by these three groups, the existing empirical studies have used factors related to systematic and diversifiable risk in order to explain the accrual anomaly. Despite previous attempts at various risk-based explanations for the accrual effect, the issue has not been completely resolved in the literature. This paper explores whether individual-assets' tail risk is related to the accrual effect, and empirically tests whether that effect is a result of market mispricing or of risk fundamentals.

Recent empirical evidence suggests that investors are not only concerned with the average variability and covariance of their assets, but are also paying attention to the tails of their assets' return distribution (Bollerslev and Todorov 2011; Embrechts et al. 1997; Xiong et al. 2014). A measure of maximum likely loss that captures the tail risk of return distribution can provide a characterization of underlying assets' risk dynamics during extreme economic uncertainty and high financial stress (Kelly and Jiang 2014). In this paper, we use a parsimonious measure of maximum likely loss known as value-atrisk (VaR) and investigate its possible contribution to the anomalous relationship between accounting accruals and the cross-section of expected stock returns. Specifically, we evaluate the relative importance of individual-assets tail risk-as opposed to aggregate tail risk or systematic risk measures that are common to many assets-in the return dynamics of accrual-sorted portfolios.

The basic idea behind a risk-based explanation for any anomalous behavior hinges on the core belief that financial assets that yield predictably higher returns are necessarily more risky. Thus, in a standard multifactor asset pricing framework, assets with higher (lower) average returns need to reflect a compensation for higher (lower) systematic risk. In such settings, any pattern that emerges in the average returns of portfolios formed using firm-level accruals should match the loadings on priced risk factors. However, it is well known that the betas associated with traditional market risk and common risk factors fail to explain the cross-section of returns of accrual-sorted portfolios. More specifically, the core idea of Sloan (1996) - that firms with high reported accruals in a given fiscal period tend to underperform firms with low reported accruals - is not explained by the Fama-French (FF) five-factors (Fama and French 2016), the gross profitability factor (Novy-Marx 2013), or the q-factor (Hou et al. 2015). In a separate strand of research, the measure of maximum likely loss has been used in the context of both general cross-sectional returns of individual stocks (Bali and Cakici 2004) and certain well-known asset pricing anomalies (Almeida et al. 2017; Aboura and Arisoy 2019). In the literature, a measure that proxies maximum likely loss or tail risk at the individual asset level has not yet been considered in the context of the anomalous relationship between accounting accruals and the expected stock returns. ${ }^{2}$

\footnotetext{
1 An incomplete list of recent works on the accrual anomaly includes Ali and Gurun (2009), Detzel et al. (2018), Fama and French (2008, 2016), Gu (2020), Hirshleifer and Jiang (2010), Hirshleifer et al. (2011), Hou et al. (2020), Khan (2008), Lewellen and Resutek (2016), Ohlson and Bilinski (2015) and Stambaugh et al. (2012).

2 A list of recent works on tail-risk that are related to this study includes Andersen et al. (2020), Bali et al. (2014), Bollerslev and Todorov (2011), Bollerslev et al. (2015), Chapman et al. (2018), Daniel et al. (2012), Kelly and Jiang (2014) and Van Oordt and Zhou (2016).
} 
Thus, one of our key objectives is to test whether individual assets' VaR explains the crosssectional differences in expected returns of portfolios sorted by accrual characteristics.

We employ a research design closely related to recent advances in the empirical corporate investment literature. We use the NYSE-AMEX-NASDAQ universe of firms between 1971 and 2017 and follow the balance sheet approach of Sloan (1996) to calculate the accrual component of earnings. We utilize dynamic time-series models that jointly parameterize conditional means and conditional variances and obtain estimates of the maximum possible loss in an asset (or a portfolio of assets) over a given time period for a given confidence level. We follow Engle and Manganelli $(2001,2004)$ and obtain our primary VaR estimates based on semiparametric GARCH predictions where the $100 \tau \%$ VaR is computed as the estimated GARCH variance times the $\tau$ empirical quantile of the standardized residuals. Among other features, the semiparametric GARCH allows the tails to reflect both the nonnormality and the dynamic behavior of the asset distribution. As a benchmark, we also estimate individual assets' VaR using standard normal-GARCH. ${ }^{3}$ This set up allows us to create various one-, two-, and three-dimensional portfolios based on distinct firm-level characteristics, including total accruals and $\mathrm{VaR}$, and utilize them in our empirical tests.

Altogether, we report three broad conclusions. The first is that the maximum likely loss plays a contributing role in explaining the accrual premium. $\mathrm{VaR}$ is in congruence with portfolio-level accruals and captures a part of the common variation appearing in accrualsorted portfolio returns. Second, there is a significant positive relationship between VaR and the cross-section of expected returns of portfolios sorted by firm-level characteristics such as size and total accruals. The widely known negative correlation between accruals and average returns does exist in our sample but decreases once we control for VaR. Finally, we show that firm-level VaR has additional explanatory power in the cross-section even after controlling for other determinants of expected returns. For all CRSP stocks, 5\% $\mathrm{VaR}$ accounts for $14.39 \%$ of the accrual premium in the absence of other characteristics, while the marginal contribution of $5 \% \mathrm{VaR}$ in the presence of other competing variables is $9.53 \%$. This finding is robust for stocks with non-NASDAQ listings. The determination that the individual-assets' tail-risk enhances our understanding of cross-sectional differences in expected returns of accrual-sorted portfolios adds to the literature.

This paper is organized as follows. The following section reviews the existing literature. The section after that describes the data and the research design. There we outline the empirical methodology used throughout the paper. The next section contains our main empirical results. It provides an overview of the characteristics of portfolios sorted by accruals, VaR, and firm size. This section also contains our regression results concerning various tests of mispricing. In the final section, we conclude with brief comments.

\footnotetext{
${ }^{3}$ Note that, the volatility measure based on the conditional standard deviation of asset returns using historical volatility model is widely recognized in the literature for its superior out-of-sample performance (Hansen and Lunde 2005; Bams et al. 2017). In practice, we can estimate the unobserved volatility of an asset using its prices, or derivatives, or both. Thus, we can also use prices from option markets and the Black-Scholes pricing formula to calculate the volatility of an asset. However, as mentioned by Neely (2009) and Tsay (2013), such a model-dependent measure of implied volatility is based on various restrictive assumptions and tends be larger than that obtained by the regression-based volatility measure. The ability of option-implied volatility measure can also be limited due to the risk premium embedded in implied volatilities (Bams et al. 2017).
} 


\section{Related literature and our incremental contributions}

The Financial Accounting Standards Board's (FASB 1978) Statement of Financial Accounting Concepts 1 states that the main objective of business financial reporting is to provide information that is useful to investors and lenders. More specifically, financial accounting information should be helpful in determining a company's cash flows, assets, liabilities, owner's equity, and changes in them (FASB 1978). Thus, a misunderstanding of financial statements related to accounting earnings and cash flows clearly goes against the main objective of financial reporting. Sloan's (1996) seminal work on the accrual effect highlighted the importance of accounting accruals and pointed out that the literature up to that point had failed to recognize that accruals were less persistent than cash flows. Since the work of Sloan (1996), numerous studies provided evidence that firms with high reported accruals in a given fiscal period tend to underperform firms with low reported accruals.

Despite considerable research, the academic investigation of the persistence and magnitude of the accrual effect is still alive two decades later. While the claim that low-accrual stocks produce superior returns compared to high-accrual stocks continues to hold true in both the US and around the world (Papanastasopoulos 2014; Pincus et al. 2007), the debate over the interpretation of this accrual premium is far from over. ${ }^{4}$ The consensus is that while the magnitude of abnormal returns has shrunk, commonly used risk factors have limited success in explaining the accrual premium, which continues to remain statistically significant (Stambaugh and Yuan 2017). Dopuch et al. (2010) investigate the sources of differential accrual pricing across profit and loss firms and find that the extent of accrual overpricing is less severe for loss-making firms than for profit-making firms. Numerous studies (see e.g., Dechow and Dichev 2002; Richardson et al. 2005) link the existence of the accrual premium with subjectivity involved in the estimation of accruals. Among others, one of the primary reasons why such subjectivity emerges is accounting distortions arising from managers' opportunistic use of accruals. If investors misunderstand the accounting distortions, it could result in the mispricing of firms with high accruals relative to those of low accruals. ${ }^{5}$ Báez-Díaz and Alam (2013) decompose total accruals into book and tax accruals and find that tax accruals are mispriced more than book accruals because of the complexity of tax accruals in assessing future earnings. Recently, Ball et al. (2016) show that a cash-based operating profitability measure is more informative about future stock returns while accrual-based earnings measure is better at capturing current period performance.

Several previous studies provided evidence for the ability of a variety of risk factors to predict cross-sectional variation in asset returns related to accruals. For example, Khan (2008) proposes a four-factor asset-pricing model that captures rationally priced economic and financial distress characteristics. Khan (2008) shows that a portion of the cross-sectional variation in returns to high- and low-accrual firms reflect a rational premium for risk. Ohlson and Bilinski (2015) use a set of logistic regressions predicting high and low returns

\footnotetext{
${ }^{4}$ Some work in the literature shows that the accrual premium is not only persistent over time but also can be observed among even more sophisticated investors (Bradshaw et al. 2001; Barth and Hutton 2004; Teoh and Wong 2001; Richardson 2003). Research has shown that the accrual premium is linked with various fundamental-to-price ratios (Bartov and Kim 2004; Desai et al. 2004).

5 For example, Doukakis and Papanastasopoulos (2014) focus on the UK and find that the accrual premium is explained by the presence of accounting distortions.
} 
to distinguish between risk and anomaly interpretations and show that accruals increase the likelihood of low returns and reduce the probability of high returns. In a related work, Hirshleifer et al. (2012) extend the FF framework by incorporating an accrual-factor-mimicking portfolio and cast doubt on the risk-based explanation. Some of the existing studies have also, with different emphases, investigated the relationship between idiosyncratic risk and the accrual effect. One such work is by Mashruwala et al. (2006) who argue that the accrual premium is concentrated in firms with high idiosyncratic return volatility; thus for a risk-averse investor it becomes very risky to take positions in stocks with extreme accruals. Some other works relate the accrual anomaly with financial distress. It has been argued that abnormal returns of accrual-sorted portfolios may result from high distress-risk exposures. For example, Avramov et al. (2013) examine the implications of financial distress in nine different asset-pricing anomalies and found that financial distress acts as a conditioning variable and explains eight of the anomalies. Furthermore, Avramov et al. (2013) argue that the accrual effect emerges from different economic premises, is insensitive to credit conditions, and is therefore an exception.

A separate strand of literature has devoted attention to the measure of tail risk-which include alternative VaR measures-in predicting a variety of financial outcomes including expected stock returns as well as future economic conditions. In the literature, tail risk measures are developed both at the individual stock level and at the aggregate level. For example, Bali et al. (2014) use a stock-level measure of hybrid tail covariance risk (HTCR) and find a positive relationship between HTCR and expected stock returns. ${ }^{6}$ Kelly and Jiang (2014) use aggregate tail risk (ATR) and show its predictive power for real economic activity and aggregate market returns. Chapman et al. (2018) find that ATR explains the cross-section of the discount rate component of returns, but not the cash-flow component. Other works that argue that tail risk can play an important role in explaining risk premiums of equities include Allen et al. (2012) and Andersen et al. (2020). ${ }^{7}$ Recently, Aboura and Arisoy (2019) examine the size, value, momentum and idiosyncratic volatility anomalies by using both HTCR and ATR. They find that HTCR explains the size premium and ATR explains the idiosyncratic volatility premium, but neither of these tail-risk measures explain the value and momentum premiums.

Three distinct features of our study separate it from existing works in the literature. First, we address the modelling of two aspects of maximum likely loss - the conditional mean and conditional volatility of asset returns, and the nonnormality of the return distribution. As we know, asset return distribution varies over time and precise estimates of time-varying moments are critical ingredients of a tail-risk measurement that characterizes such return distributions. For this reason, we use dynamic models, which jointly parameterize conditional means and conditional variances, in conjunction with a semiparametric method, which is robust to nonnormality, to provide a better measure of VaR. Second, we use long time-series of individual assets to estimate $\mathrm{VaR}$, which varies across assets. Thus, the estimate of $\mathrm{VaR}$ provides useful characterization of investment risk-especially during extremely volatile periods and crises. Third, the fact that the estimate of VaR has wide cross-sectional variation allows us to test whether individual assets sorted on different

\footnotetext{
6 In a related paper, Bali and Cakici (2004) examine the empirical performance of VaR in capturing crosssectional and time-series variation in stock returns. Their results suggest that VaR explains cross-sectional variation in expected returns both at the stock level and at the portfolio level.

7 It has also been suggested that there is a significant cross-sectional relation between hedge fund returns and VaR (Bali et al. 2007; Agarwal et al. 2017).
} 
firm-level characteristics have different exposures to $\mathrm{VaR}$. In fact, we find that our individual assets' tail-risk measure is a significant determinant of expected returns of accrualsorted portfolios in the cross-section. Thus, compared to existing measures such as HTCR and ATR, our tail-risk measure is distinct and demonstrates unique individual asset-level risk characterization. ${ }^{8}$

In essence, unlike FF risk factors, which capture risk common to many assets, the individual assets' maximum likely loss captures risk that can potentially be caused by extreme events such as the 2007-2009 financial crisis, a corporate bankruptcy, the collapse of a company, or the recent coronavirus pandemic. Compared to prior research, we highlight the importance of the connection between these maximum likely losses, as proxied by $\mathrm{VaR}$, and the mispricing of the accrual component of earnings. Taken together, our results provide a support for the mispricing explanation of the accrual anomaly. In the absence of firm characteristics, the accrual- and VaR-based risk factor loadings are compensated with higher expected returns. However, when we augment risk factors with accruals and VaR, those firm-level characteristics subsume the role of those risk factors, a result that undercuts the validity of the market risk explanation while confirming the market-mispricing hypothesis.

\section{Data, variable measurement, and test portfolio construction}

In this section, we briefly describe the data and variables used throughout the paper. Then we outline the construction of various test portfolios based on the described variables and how we implement a standard research design. As in most studies, our sample includes all US firms with nonmissing size and accrual information listed on NYSE-AMEX-NASDAQ with monthly return data on the CRSP and annual accounting data on Compustat. We exclude firms with negative book value, financial firms, and we apply a price screen of $\$ 1$. Our sample period is between January 1971 and December 2017. Following the convention of the existing literature, we ensure that the accounting data is available before we examine stock returns by matching the accounting information for all fiscal year ends in calendar year $t-1$ with the stock returns from July of year $t$ to June of year $t+1$. We define size as the natural logarithm of CRSP market value for June of year t, book equity (BE) as the Compustat book value for fiscal year end (Compustat data item 60) in calendar year $t-1$, and book-to-market (BE/ME) as the ratio of book equity and market capitalization at the end of year $\mathrm{t}-1$.

For accruals, we use the balance sheet and income statement approach and calculate operating accruals by the change in non-cash working capital less depreciation expense deflated by average total assets. Following Sloan (1996), we obtain the accrual component of earnings by

\footnotetext{
${ }^{8}$ It is important to note that we don't intend to conduct a horse race between alternative measures of tailrisk. Instead, our focus is to test the explanatory power of individual assets' tail-risk in the context of the accrual anomaly.
} 


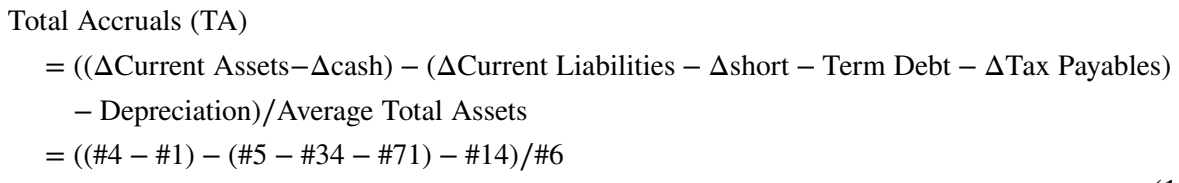

where $\Delta$ denotes a one-period backward difference and \#'s indicates Compustat items. ${ }^{9}$ We also use the firm-level earnings (Compustat data item 178) plus depreciation (Compustat data item 14) minus working capital accruals and obtain the operating cash-flow-to-price ratio $(\mathrm{CFO} / \mathrm{P})$. We include only firms that have appeared on Compustat for at least two years. Due to insufficient data, we eliminate pre-1971 observations. Altogether, our final sample yields 84,706 firm-year observations with December fiscal-year ends from 1971 to 2017. As is known in the literature (see e.g., Sloan 1996; Desai et al. 2004), aligning firms in calendar time by using December fiscal-year-end firms allows the estimation of profitability on accrual mispricing. We obtain the FF factors from Ken French and the bond yields from the Federal Reserve Bank of St. Louis. The set of FF factors include a traditional market factor (Mktrf), a size factor $(S M B)$, a value factor $(H M L)$, an investment factor $(C M A)$, and a profitability factor $(R M W) .^{10}$

In the literature, many existing works highlight the benefits of the general GARCH-type model as a benchmark for VaR forecasting (Giacomini and Komunjer 2005; Gerlach et al. 2011). In this paper, in order to quantify the maximum likely loss empirically, we consider the estimation of VaR at the individual asset level within the GARCH-type framework that jointly parameterizes conditional means and conditional variances. We create two measures of VaR - a primary measure of semiparametric VaR estimates using Quasimaximum likelihood (QML)-GARCH predictions, and a benchmark measure of parametric VaR estimates based on ML-GARCH predictions under standard normal distributions. For both approaches, we use an $\mathrm{AR}(1)$ specification for the conditional mean and a $\operatorname{GARCH}(1,1)$ specification for conditional variance of each asset. Thus, for a time-series of $R_{i, t}(i=1, \ldots N, t=1, \ldots T)$, our parsimonious specification takes the following form

$$
\begin{aligned}
R_{i, t} & =\mu_{i, t}+\eta_{t}, \quad \mu_{i, t}=\alpha_{0}+\alpha_{1} R_{i, t-1} \\
\eta_{t} & =\sqrt{h_{i, t}} \varepsilon_{i, t}, \quad \varepsilon_{i, t} \sim \operatorname{iid}(0,1), \quad h_{i, t}=\delta_{0}+\delta_{1} \eta_{t-1}^{2}+\delta_{2} h_{i, t-1}
\end{aligned}
$$

where $\mu_{i, t}$ and $\sqrt{h_{i, t}}$ are the conditional mean and conditional volatility for $R_{i, t}$. For the parametric method, we estimate the parameters of the $\operatorname{AR}(1)+\operatorname{GARCH}(1,1)$ model $(2)$ by ML under the assumption of normality, and obtain the $100 \tau \%$ VaR at time $t$ by

$$
\operatorname{VaR}_{t}(\tau)=\widehat{\mu}_{i, t}+\sqrt{\widehat{h}_{i, t}} Q_{\varepsilon}(\tau)
$$

\footnotetext{
9 The makeup of our sample is guided by a desire to compare our results with past studies. We also use beginning-of-the-year total assets as the deflator in (1). Our results are not sensitive to this normalization. One natural limitation of our sample period is the possible influence of mergers and acquisitions.

${ }^{10}$ Note that, in a five-factor model, assets expected return depends on the sensitivity of its return to the market return, and four mimicking portfolios representing additional factors. Similar to Fama and French (2016), we do not report any results involving the momentum factor proposed by Carhart (1997) and the liquidity factor proposed by Pastor and Stambaugh (2003) in our asset pricing tests, as these two additional factors produce small changes in model performance but our overall message remains unchanged. Details are available upon request.
} 
where $Q_{\varepsilon}(\tau)$ denotes the unconditional $\mathrm{VaR}$ of the error term $\varepsilon_{i, t}$. For the semiparametric method, we first obtain a QML estimate of the $\operatorname{AR}(1)-\operatorname{GARCH}(1,1)$ model for each individual asset returns and then compute each assets' $100 \tau \%$ VaR from (3) by multiplying the empirical quantile of the standardized residuals by $\sqrt{\widehat{h}_{i, t}}$.

The QML-GARCH model is proposed by Engle and Manganelli $(2001,2004)$ and based on the results of Bollerslev and Woolridge (1992), who show that, even if the standardized residuals do not come from a normal distribution, the ML estimates of GARCH parameters are consistent, provided the variance equation is correctly specified. ${ }^{11}$ While the use of the ML method under normality is relatively simple and widely known, an appealing feature of the joint estimation of conditional mean and variance under the QML method is its robustness to nonnormality. In addition, as mentioned by Engle and Manganelli (2001), the QML-GARCH requires that the tails have the same dynamic behavior as the rest of the asset distribution. It is noteworthy that both of the VaR estimates are measured monthly, due to the availability of long historical data for individual stocks and portfolio returns. Further details on VaR construction and underlying methodology is given in the "Appendix".

We utilize the individual measures of firm-level characteristics as well as the VaR estimate to construct various one-, two-, and three-dimensional portfolios. Our first set of onedimensional test portfolios utilize univariate sorts on TA deciles. To construct such portfolios, in December of each year we create NYSE decile breakpoints based on individual firms' TA measures from (1). Then, using those NYSE breakpoints for TA, we allocate all CRSP stocks into ten accrual portfolios and compute the value-weighted monthly returns on these portfolios. Our set of second one-dimensional test portfolios is based on univariate sorts of VaR deciles. First, we use each individual NYSE firm's VaR estimate and create decile breakpoints in December of each year. Then, using those NYSE breakpoints, we allocate all CRSP stocks into VaR deciles and compute the corresponding value-weighted monthly returns. Thus, the first $\mathrm{VaR}$ decile consists of the $10 \%$ of all stocks that have the lowest VaR, and the last VaR decile consists of the $10 \%$ of all stocks with the highest VaR.

We also create two sets of multidimensional portfolios that are double- and triple-sorted using distinct firm-level characteristics. These sets of multidimensional test portfolios consist a set of nine portfolios based on TA and VaR, and a set of twenty-seven portfolios based on size, TA and VaR. For two-dimensional portfolios, the bivariate sorts using NYSE breakpoints are conducted independently, and test portfolios are formed in December of each year. Thus, for the nine portfolios based on TA and VaR, all stocks are sorted independently into three TA groups using the bottom $30 \%$, middle $40 \%$, and top $30 \%$ of break points for NYSE firms. The same sample of stocks are also sorted independently into three VaR groups using the bottom $30 \%$, middle $40 \%$, and top $30 \%$ of break points for NYSE firms. Consequently, nine portfolios are formed at the intersections of the three TA groups and three VaR groups. For the triple-sorted portfolios, all CRSP stocks are first sorted independently into three size groups using the bottom 30\%, middle $40 \%$, and top $30 \%$ of break points for NYSE firms. The same sample of all CRSP stocks are also sorted independently

\footnotetext{
11 We also use the CAViaR method of Engle and Manganelli (2004) to estimate the individual assets' VaR. The corresponding results do not provide any improvement over those reported in this paper. This finding is not entirely surprising. For example, Giacomini and Komunjer (2005) suggest that, at the 1\% quantile level, the CAViaR method is the most efficient method but at the 5\% quantile level, the GARCH model with normal distribution is better than CAViaR. In a related work, Gerlach et al. (2011) find similar results across a range of financial market indices.
} 
into three TA groups and three VaR groups using the respective bottom 30\%, middle $40 \%$, and top 30\% of break points for NYSE firms. Consequently, twenty-seven portfolios are formed at the intersections of the three size groups, three TA groups and three VaR groups. All reported results on multidimensional test portfolios are based on semiparametric VaR estimates using $\mathrm{AR}(1)-\mathrm{GARCH}(1,1)$ predictions. All value-weighted monthly returns are in excess of the one-month T-bill rate.

The idea behind this portfolio construction scheme is to ensure that the test portfolios differ in their level of TA and size, and that there is a wide range of average returns in our testing assets. The research approach adopted in this paper is consistent with much of the prior literature. Unlike the firm-level data, the portfolio-level data help to mitigate concerns such as infrequent trading and outliers. In the next section, we analyze the aforementioned sets of portfolios for empirical analysis.

\section{Empirical results}

\subsection{Characteristics of one-dimensional portfolios}

We start with a close look at our first set of testing portfolios, which are formed by univariate sorting of stocks using TA deciles, as mentioned in Sect. 3. Table 1 provides the valueweighted average monthly returns and other selected features of accrual deciles. In terms of the overall spread, the average portfolio returns range from $1.29 \%$ per month for the lowest-TA decile to $0.82 \%$ per month for the highest-TA decile, moving in a monotonically decreasing pattern. The low-minus-high TA portfolio, or the accrual-based zero-investment portfolio, generates a statistically significant premium of $0.47 \%$ per month. ${ }^{12}$ Similar to prior work, the average measure of accruals reported in column three indicates that all six bottom decile portfolios having negative TA. The following four columns provide information on average size and BE/ME characteristics for all portfolios. Together, they suggest that, on average, the extreme TA portfolios consist of small-size firms, and portfolios adjacent to the median TA decile tend to contain big-size firms. Furthermore, portfolios with lower TA (higher TA) display a higher (lower) BE/ME ratio and thus consist of value (growth) firms. Although the average BE/ME decreases as we move from low- to high-TA portfolios, the average size does not display any such monotonic pattern.

In columns (8) through (10), we report three different estimates of semiparametric VaR for separate confidence levels. For all ten portfolios, the VaR estimates range from 14.62 to 9.38 at the $1 \%$ level, 11.70 to 6.91 at the 5\% level, and 9.29 to 5.04 at the $10 \%$ level. The lowest TA decile always displays the highest VaR estimates, which then monotonically decrease up to the fifth decile and further increase through the highest deciles. For all the confidence levels, the lowest decile continues to display slightly higher VaR than the highest decile portfolios. In the following three columns-(11) through (13)—we report the parametric VaR estimates. A high concentration of tail-risk among extreme TA deciles is also visible when we use this alternative VaR measure. Interestingly, three middle deciles

\footnotetext{
12 We note that our Table 1 result, which is for the entire sample, doesn't match with the results of Green et al. (2012), who shows a decline in the returns in the later years in the sample. However, compared to prior research, Green et al. (2012) use Compustat Point-in-Time data to replicate the nature and timing of accruals information. Thus, it is possible that the findings of Green et al. (2012) is due to their sample period or methodology specific and more study needs to be done before arriving at any conclusion.
} 


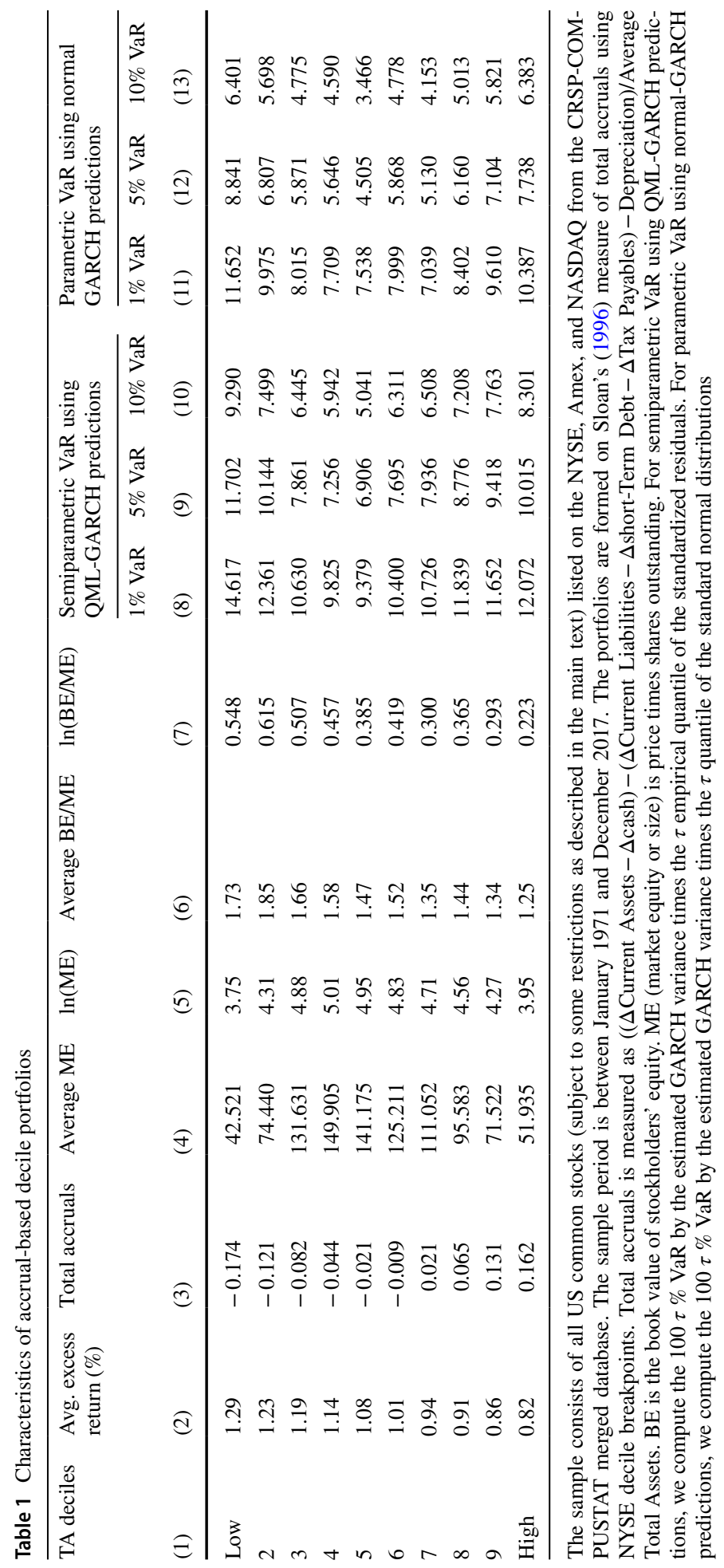


(i.e., fourth through sixth) always contain the lowest VaR estimates. Despite the difference in magnitudes, the pattern of $\mathrm{VaR}$ is consistent in the last three columns.

Thus, our first reported results confirm the findings of the prior literature that lowaccrual firms have higher average returns than high-accrual firms (Fama and French 2008), and that low-accrual firms tend to be smaller in size (Khan 2008) and display features of value firms (Hirshleifer et al. 2012). Furthermore, the results also reveal that portfolios with lower accruals display relatively higher estimates of VaR in our sample. Thus, it is reasonable to expect that the accrual premium is linked to not only firm size and fundamental-to-price ratios such as BE/ME but also to individual portfolios' tail-risk.

An obvious question is whether one-dimensional TA-sorted portfolios exhibit features that are also shared by VaR-sorted portfolios. In Table 2, we investigate that issue and report certain characteristics of our VaR-sorted portfolios. In this univariate sort, decile 1 represents the $10 \%$ of all CRSP stocks with the lowest estimated 5\% VaR, and decile 10 represents the $10 \%$ of all CRSP stocks with the highest estimated $5 \%$ VaR, etc.

The evidence presented in Table 2 suggests that the portfolios with higher (lower) VaR consistently generate higher (lower) average returns, and the average monthly returns increase monotonically from low- to high-VaR deciles. For the QML-GARCH VaRbased portfolios, the average excess returns for the highest and lowest deciles are $1.28 \%$ and $0.87 \%$ per month respectively. On average, TA decreases monotonically from low- to high-VaR deciles with low-VaR portfolios displaying positive TA and high-VaR portfolios displaying negative TA. The average firm size increases from the lowest to the fifth VaR deciles and then decreases through the highest-VaR deciles. The average BE/ME, on the other hand, monotonically increases over low- to high-VaR deciles.

The next columns show that a sorting based on normal-GARCH VaR also portrays a similar picture. In general, portfolios with higher VaR have higher returns. The highest decile earns $1.19 \%$ per month while the lowest decile earns $0.84 \%$ per month, and both are significant at the $1 \%$ level. The general finding is that normal-GARCH predictions slightly underestimate the $\mathrm{VaR}$, although it allows a complete characterization of the distribution of individual asset returns.

For both types ofVaR-sorted portfolios, the summary of average returns demonstrates the presence of a statistically significant VaR premium. ${ }^{13}$ The high-minus-low portfolio, which holds the highest 5\% VaR decile and shorts the lowest 5\% VaR decile, generates a premium of $0.41 \%$ per month for QML-GARCH and $0.35 \%$ per month for normalGARCH. ${ }^{14}$ To investigate whether the returns to the accrual-based zero-investment portfolio partially mimics the return of a similar VaR-based portfolio, we look at the correlation between the corresponding long-short portfolios. The estimated correlation is small $(0.152)$ but statistically significant (with $p$ value $<0.01$ ). The small positive correlation suggests that the accrual effect can be associated with the VaR effect but there is no indication of a causality between their long-short portfolios, or that VaR is the only risk that can explain the accrual effect.

In essence, although the constituents of the two sets of one-dimensional portfolios in Tables 1 and 2 are different, there are similarities in average returns and VaR estimates. The evidence implicitly suggests that tail-risk characteristics appear to be in congruence

\footnotetext{
13 It is important to note that in our sample the size premium is statistically insignificant $0.27 \%$ per month whereas the value premium is $0.53 \%$ per month, which is statistically distinguishable from zero.

14 In comparison, the high-minus-low portfolio corresponding to $1 \%$ VaR generates a slightly lower premium of $0.36 \%$ per month for the QML-GARCH and $0.31 \%$ per month for the normal-GARCH.
} 


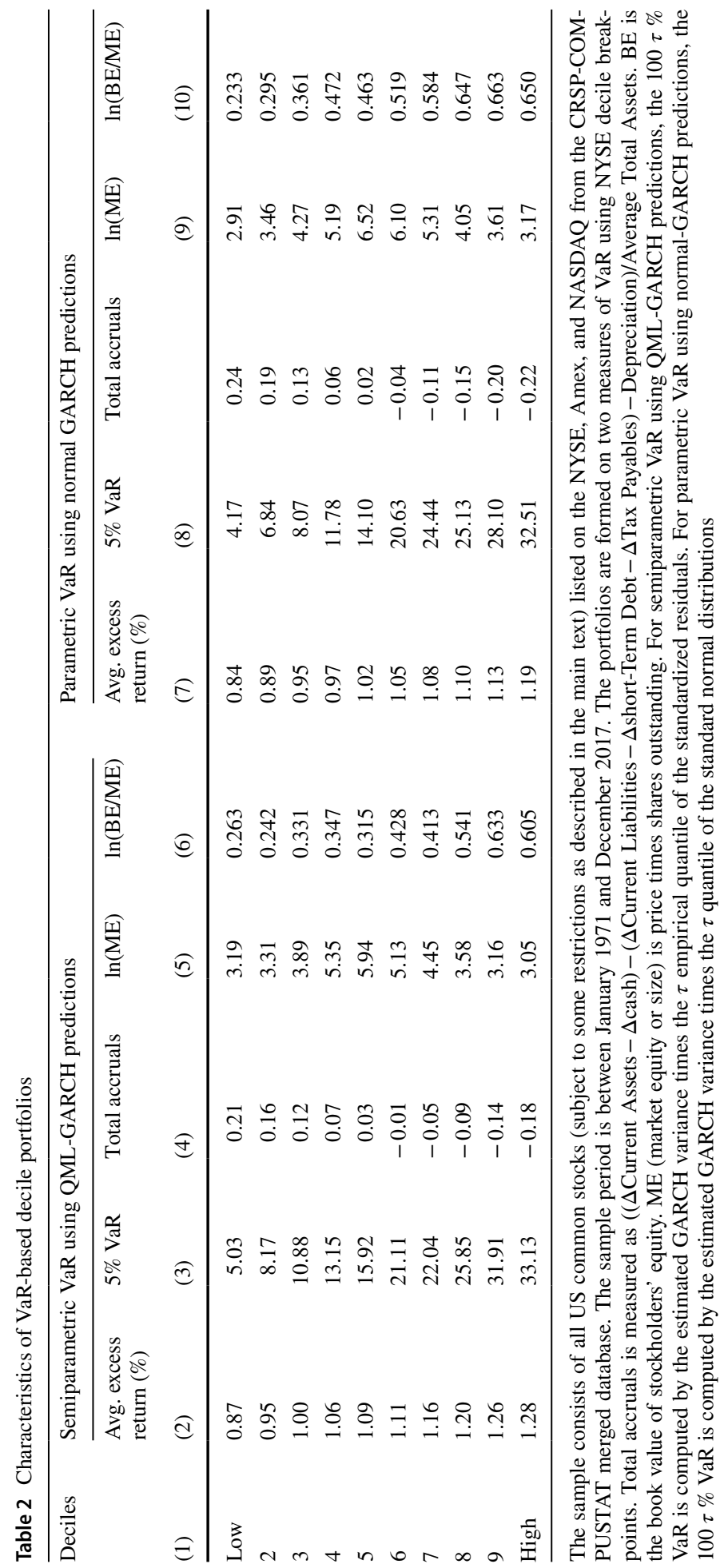


with portfolio-level accruals and may possibly explain a part of the return variability of accrual-sorted portfolios. These univariate analyses, however, do not suggest that tail-risk is the only, let alone dominant, characteristics that is associated with the accrual effect. The above evidence also raises an obvious question: Does tail-risk indeed explain the crosssectional return variation of accrual-sorted portfolios? Intuitively, if the accrual premium is not driven by individual assets' tail-risk, the spread between the low- and high-TA portfolios should be unaffected in the cross-section even after controlling for VaR. On the other hand, if individual-assets tail-risk has an influence on the returns of accrual-sorted portfolios, the spread between the low- and high-TA portfolios after controlling for VaR should weaken. In the next subsection, we investigate that presumption.

\subsection{Characteristics of two-dimensional portfolios}

In Table 3, we examine the average performance of three sets of two-dimensional portfolios using three alternative double-sorting schemes. All test portfolios are formed annually using NYSE breakpoints of TA and semiparametric VaR. For the first set, we sort all stocks into three terciles of TA (i.e., low, medium, and high), all of which are then sorted into three terciles of VaR (i.e., low, medium, and high). We then compute the value-weighted average excess returns of the resulting nine double-sorted portfolios. Panel A shows the result. For the second set of portfolios, reported in Panel B, we reverse the sorting order. Here, all stocks are first sorted into three VaR groups, which are then further sorted into three TA subgroups. In the third set of two-dimensional portfolios, all stocks are doublesorted independently by TA and VaR, and simultaneously placed into nine subgroups of TA and VaR. Panel $\mathrm{C}$ reports the average excess returns of this third set of portfolios. For each bivariate set, we rebalance each portfolio annually and hold the positions over 12 months.

The results in Panel A show that a double-sorting based first on TA and then on 5\% VaR leads to a wide range of average returns, from 0.90 to $1.41 \%$ per month. Across all three VaR terciles, the average return decreases from low- to high-TA portfolios. Similarly, in all TA terciles, the average return increases from low- to high-VaR portfolios. Thus, the bivariate sorting also confirms that there is a negative relationship between TA and average returns, and a positive relationship between $\mathrm{VaR}$ and average returns. In terms of the overall performance, the portfolio with low-TA/high-VaR generates the highest average return, while the high-TA/low-VaR portfolio generates the lowest average return. The differences between average returns for high- and low-VaR portfolios range from 0.10 to $0.28 \%$ per month, although the VaR premium is statistically significant at the $1 \%$ level only for lowTA subgroups. The TA-based long-short portfolios generate an average return of $0.24 \%$ for the low-VaR and $0.40 \%$ for the high-VaR subgroups. The accrual premium is statistically significant across all three VaR subgroups. The results corresponding to bivariate sorts based on TA and $1 \%$ VaR remain consistent.

In Panel B, after we reverse the sorting order, there are small but interesting changes in the overall pattern of average returns. The portfolios within the low-TA as well as high-VaR groups continue to generate the highest average returns. For the low-TA group, the average return increases from $1.10 \%$ per month for the low-VaR tercile to $1.28 \%$ per month for the high-VaR tercile. In contrast, the portfolios within the high-TA group show a monthly return of $0.94 \%$ for the low-VaR tercile and $1.08 \%$ for the high-VaR tercile. The three highVaR portfolios generate an average return of $1.20 \%$ per month, while the three low-VaR portfolios generate an average return of $1.04 \%$ per month. The return on the VaR-based 


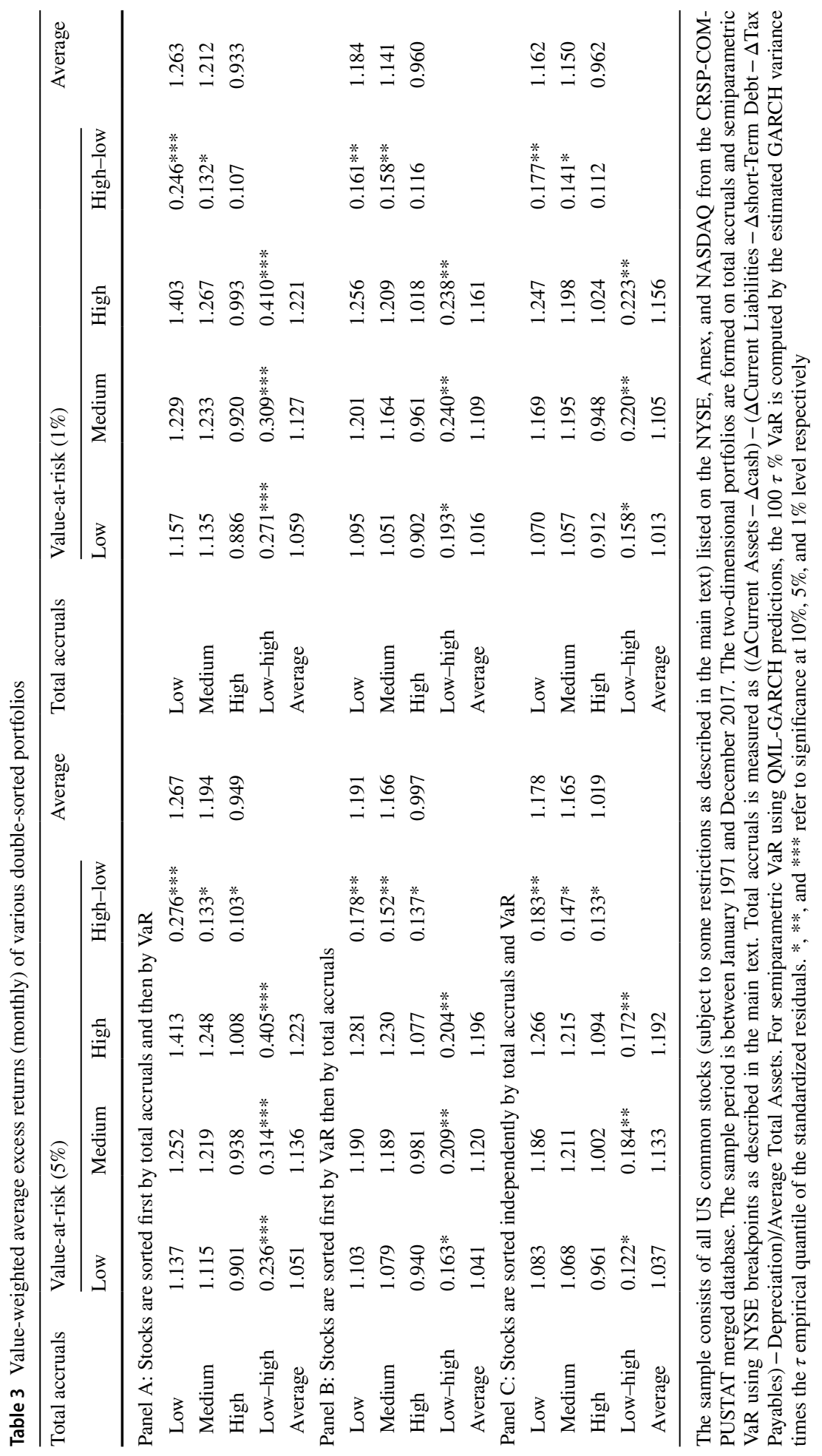


long-short portfolio is highest $(0.18 \%$ per month) for the low-TA category and lowest $(0.14 \%)$ for the high-TA category. The main surprise of this reverse double sorting in Panel B is the dispersion of accrual premiums over the three VaR groups. Here, when all stocks are sorted first by $5 \%$ VaR and then by TA, the average spread between the low- and high-TA portfolio increases from $0.16 \%$ for low-VaR to $0.21 \%$ for medium-VaR, but then decreases slightly to $0.20 \%$ for the high-VaR tercile. When we focus on the $1 \%$ VaR-related results, the resulting portfolio generates an average monthly accrual premium of $0.19 \%$ for the low-VaR, and around $0.24 \%$ for both medium- and high-VaR terciles. Thus, unlike in Panel A, the relationship between the average returns of TA-based long-short portfolios and $\mathrm{VaR}$ is less consistent in Panel B.

In Panel C, where stocks are double-sorted independently, the overall pattern in average returns remain consistent with the previous panel, although there are slight changes in the spread of both VaR and accrual premiums. For 5\% VaR-based portfolios, the average return of the low-TA group is now lower (1.18\% in Panel C vs. $1.27 \%$ in Panel A), and that of the high-TA group is now slightly higher (1.02\% in Panel C vs. $0.95 \%$ in Panel A). On average, portfolios corresponding to the low-TA tercile continue to generate higher returns compared to their high-TA counterparts, but the differences are no longer as large as in Panel A. The average return of the three VaR groups are not discernibly different, although the average spread in returns between high- and low-VaR portfolios decreases marginally from the low- to high-TA terciles. If we shift our focus to $1 \%$ VaR-related results, the lowVaR tercile yields an average accrual premium of $0.16 \%$ per month, and both the mediumand high-VaR terciles generate an average accrual premium of $0.22 \%$ per month. Thus, we find that the spreads between low- and high-TA portfolios after controlling for VaR are significantly attenuated. Heuristically, the framework also allows us to construct long-short portfolios for mimicking the risk factors in average returns related to TA and VaR.

It is important to recognize that so far, we have used only TA- and VaR-related sorts to construct portfolios. In the literature, it is well known that the value-weighted portfolio returns from sorts based on variables other than firm size can be influenced by the dominance of big stocks. There is a serious concern associated with firm size in any portfolio sorting based on individual asset-level characteristics, and thus it is important to consider other dimensions of portfolio sorts besides accruals in our testing assets. In the subsection that follows, we do so.

\subsection{Characteristics of three-dimensional portfolios}

In Table 4, we report the average performance of our three-dimensional portfolios. Here, all CRSP stocks are sorted independently by firm size, TA and 5\% VaR, and simultaneously placed into twenty-seven subgroups. The three-way sorts help us to examine the accrual effect after controlling for size and semiparametric VaR.

Several results are notable. In terms of overall performance, portfolios with smaller size, lower TA and higher VaR generate higher average returns, while portfolios with bigger size, higher TA and lower VaR generate lower average returns. As we scan through the size groups, the average returns decrease from low- to high-TA groups and increase from lowto high-VaR groups. The low-TA portfolios generate higher average monthly returns than the high-TA portfolios ( $1.20 \%$ per month vs. $0.97 \%$ per month). The triple-sorted portfolios also confirm a positive relationship between $\mathrm{VaR}$ and average returns. The sets of nine low-VaR and nine high-VaR portfolios generate an average return of $1.02 \%$ and $1.14 \%$ per month, respectively. As expected, compared to medium- and big-size, small-size portfolios 
generate higher average returns. Overall, the VaR premium is more pronounced for smallsize and low-accrual stocks, and the size premium, on the other hand, shows no discernible patterns.

The results suggest that the accrual effect, in retrospect, is not independent of the size or VaR effects and vice versa. For instance, the accrual premium increases from low- to high-VaR groups but decreases from small- to big-size groups. For any given size group, the average monthly return declines with accruals and increases with VaR groups. Among small-size group, firms with the lowest accruals earn a significant higher average return- $0.24 \%$ per month - than firms with the highest accruals. The small firms perform better than the big firms mainly in the low-accrual and high-VaR groups. Irrespective of any size group we consider, firms with low TA and high VaR earn substantially more than do firms with high TA and low VaR, and the spread between small- and big-size portfolios is statistically insignificant. The key finding-that the average spread in returns between low- and high-TA portfolios are less consistent across the VaR groups-echoes our results from Table 3. Thus, even in the presence of firm size, the individual assets' tail risk helps to disentangle the overall spread in accrual premiums, and it is reasonable to say that such tail-risk measures proxied by VaR have non-negligible predictive power in the cross-section.

\subsection{Risk-adjusted returns of the testing portfolios}

In order to understand the nature of the risk-adjusted returns of the characteristics-sorted portfolios, we evaluate the intercepts from the following time-series regression model

$$
R_{i, t+1}=\alpha_{i}+\beta_{i}^{\prime} f_{t+1}+\varepsilon_{i, t+1}, \forall i=1, \ldots, N
$$

where $R_{i, t+1}$ represents the excess returns of asset $i$ at time $t+1$, and $f_{t+1}=\left(f_{1, t+1}, \ldots, f_{K, t+1}\right)^{\prime}$ is the set of risk factors, $\beta_{i}=\left(\beta_{1}, \ldots, \beta_{K}\right)^{\prime}$ is the vector of risk factor exposure and $\alpha_{i}$ represents the risk-adjusted abnormal return of asset $i$. We use two basic factor models: a 1-factor (1F) model that uses excess market return, and a FF 5-factor (5F) model that adds size, value, investment and profitability factors. ${ }^{15}$ Each model is then reevaluated in the presence of TA- and VaR-based long-short portfolios, given by LMHA and HMLV respectively. ${ }^{16}$

Analogous to the SMB and HML, both LMHA and HMLV are constructed using the 6 value-weighted portfolios formed on TA and VaR, and represent two long-short portfolios that mimic the risk factor in average returns related to accruals and VaR, respectively. Each year, stocks are sorted independently into three TA portfolios (L, M, or H) and two VaR portfolios $(\mathrm{L}$ or $\mathrm{H})$ using their NYSE breakpoints, resulting in six new portfolios $(\mathrm{L} / \mathrm{L}$, $\mathrm{L} / \mathrm{H}, \mathrm{M} / \mathrm{L}, \mathrm{M} / \mathrm{H}, \mathrm{H} / \mathrm{L}, \mathrm{H} / \mathrm{H})$ formed at the intersections of those size and TA portfolios. LMHA is the difference between the equal-weighted average of the $\mathrm{L} / \mathrm{L}$ and $\mathrm{L} / \mathrm{H}$ portfolio returns and the equal-weighted average of the $\mathrm{H} / \mathrm{L}$ and $\mathrm{H} / \mathrm{H}$ portfolio returns. HMLV is the

\footnotetext{
15 As is well known, the size and value factors are empirically motivated and associated with the static dimensions of the firms. In contrast, the investment and profitability factors are theoretically motivated. According to the q-factor asset pricing model (Hou et al. 2015), there are two drivers of expected stock returns: the expected discounted profitability of the firm and the investment-to-asset ratio. The addition of $C M A$ and $R M W$ therefore captures the two drivers of expected returns in the q-factor model.

16 Thus, a full specification of (4) with all seven factors takes the following form: $R_{i, t+1}=\alpha_{i}+\beta_{M k t r f} M_{k t r f} f_{t+1}+\beta_{S M B} S M B_{t+1}+\beta_{H M L} H M L_{t+1}+\beta_{C M A} C M A_{t+1}+\beta_{R M W} R M W_{t+1}+\beta_{L M H A} L M H A_{t+1}+$ $\beta_{H M L V} H M L V_{t+1}+\varepsilon_{i, t+1}, \forall i=1, \ldots, N$.
} 
Table 4 Value-weighted average excess returns (monthly) of triple-sorted portfolios

\begin{tabular}{|c|c|c|c|c|c|c|}
\hline Size & Total accruals & Low & Medium & High & High-low & Average \\
\hline \multicolumn{7}{|c|}{ Value-at-risk (5\%) } \\
\hline \multirow{5}{*}{ Small } & Low & 1.141 & 1.245 & 1.332 & $0.191 * *$ & 1.239 \\
\hline & Medium & 1.051 & 1.137 & 1.152 & $0.101^{*}$ & 1.113 \\
\hline & High & 0.964 & 0.978 & 1.051 & 0.087 & 0.998 \\
\hline & Low-high & $0.177^{*}$ & $0.267 * * *$ & $0.281 * * *$ & & \\
\hline & Average & 1.052 & 1.120 & 1.178 & & \\
\hline \multirow[t]{5}{*}{ Medium } & Low & 1.126 & 1.214 & 1.302 & $0.176^{* *}$ & 1.214 \\
\hline & Medium & 1.037 & 1.105 & 1.118 & 0.081 & 1.087 \\
\hline & High & 0.920 & 0.951 & 1.027 & 0.107 & 0.966 \\
\hline & Low-high & $0.206^{*}$ & $0.263 * * *$ & $0.275^{* * *}$ & & \\
\hline & Average & 1.028 & 1.090 & 1.149 & & \\
\hline \multirow[t]{5}{*}{ Big } & Low & 1.063 & 1.182 & 1.213 & $0.150^{* *}$ & 1.153 \\
\hline & Medium & 0.996 & 1.042 & 1.091 & 0.095 & 1.043 \\
\hline & High & 0.914 & 0.951 & 0.992 & 0.078 & 0.952 \\
\hline & Low-high & 0.149 & $0.231 * *$ & $0.221 * * *$ & & \\
\hline & Average & 0.991 & 1.058 & 1.099 & & \\
\hline Total accruals & Size & Low & Medium & High & High-low & Average \\
\hline \multicolumn{7}{|c|}{ Value-at-risk (5\%) } \\
\hline \multirow[t]{5}{*}{ Low } & Small & 1.141 & 1.245 & 1.332 & $0.191 * *$ & 1.239 \\
\hline & Medium & 1.126 & 1.214 & 1.302 & $0.176^{* *}$ & 1.214 \\
\hline & Big & 1.063 & 1.182 & 1.213 & $0.150^{* *}$ & 1.153 \\
\hline & Small-big & 0.078 & 0.063 & $0.119^{*}$ & & \\
\hline & Average & 1.110 & 1.214 & 1.282 & & \\
\hline \multirow[t]{5}{*}{ Medium } & Small & 1.051 & 1.137 & 1.152 & $0.101^{*}$ & 1.113 \\
\hline & Medium & 1.037 & 1.105 & 1.118 & 0.081 & 1.087 \\
\hline & Big & 0.996 & 1.042 & 1.081 & 0.085 & 1.040 \\
\hline & Small-big & 0.055 & $0.095^{*}$ & 0.071 & & \\
\hline & Average & 1.028 & 1.095 & 1.117 & & \\
\hline \multirow[t]{5}{*}{ High } & Small & 0.964 & 0.978 & 1.051 & 0.087 & 0.998 \\
\hline & Medium & 0.920 & 0.951 & 1.027 & $0.107^{*}$ & 0.966 \\
\hline & Big & 0.914 & 0.951 & 0.992 & 0.078 & 0.952 \\
\hline & Small-big & 0.050 & 0.027 & 0.059 & & \\
\hline & Average & 0.933 & 0.960 & 1.023 & & \\
\hline
\end{tabular}

The sample consists of all US common stocks (subject to some restrictions as described in the main text) listed on the NYSE, Amex, and NASDAQ from the CRSP-COMPUSTAT merged database. The sample period is between January 1971 and December 2017. For each three-dimensional portfolio, stocks are sorted independently by firm size, total accruals and $5 \%$ VaR. For all sorts, NYSE breakpoints are used. Firm Size is price times shares outstanding. Total accruals is measured as $((\Delta$ Current Assets $-\Delta$ cash $)-(\Delta$ Current Liabilities $-\Delta$ short-Term Debt $-\Delta$ Tax Payables $)-$ Depreciation $) /$ Average Total Assets. For semiparametric VaR using QML-GARCH predictions, the $100 \tau \%$ VaR is computed by the estimated GARCH variance times the $\tau$ empirical quantile of the standardized residuals. *, **, and *** refer to significance at $10 \%, 5 \%$, and $1 \%$ level respectively 
Table 5 Risk-adjusted abnormal returns of the accruals-based decile portfolios

\begin{tabular}{|c|c|c|c|c|c|}
\hline \multirow{3}{*}{$\begin{array}{l}\text { Deciles } \\
\text { (1) }\end{array}$} & \multicolumn{2}{|c|}{$\begin{array}{l}\text { Models without LMHA } \\
\text { and HMLV }\end{array}$} & \multicolumn{3}{|c|}{ Models with LMHA or HMLV or both } \\
\hline & 1-Factor & 5-Factor & 5-Factor + LMHA & 5-Factor + HMLV & $\begin{array}{l}\text { 5-Fac- } \\
\text { tor + LMHA + HMLV }\end{array}$ \\
\hline & (2) & (3) & (4) & (5) & (6) \\
\hline Low & $0.23 * * *$ & $0.20 * * *$ & $0.18^{* *}$ & $0.21 * * *$ & $0.15^{* *}$ \\
\hline 2 & $0.21 * * *$ & 0.17 *** & $0.15^{* * *}$ & $0.16^{* * * *}$ & $0.12 * *$ \\
\hline 3 & $0.15^{* *}$ & $0.14 * *$ & $0.10 * *$ & $0.12 * *$ & $0.09 *$ \\
\hline 4 & $0.14 * *$ & $0.10 *$ & $0.07 *$ & $0.08 *$ & 0.06 \\
\hline 5 & $0.10^{*}$ & 0.07 & 0.04 & 0.05 & 0.03 \\
\hline 6 & $0.09 *$ & 0.05 & 0.02 & 0.04 & -0.05 \\
\hline 7 & 0.06 & $-0.09 *$ & $-0.08 *$ & $-0.11^{* *}$ & $-0.07 *$ \\
\hline 8 & $0.10^{*}$ & -0.12 & $-0.07 *$ & $-0.09 *$ & $-0.08^{*}$ \\
\hline 9 & $-0.19 * * *$ & $-0.16^{* * *}$ & $-0.13 * *$ & $-0.15^{* *}$ & $-0.10 * *$ \\
\hline High & $-0.25^{* * *}$ & $-0.22 * * *$ & $-0.17 * *$ & $-0.19 * *$ & $-0.14 * *$ \\
\hline Long-short & $0.48 * * *$ & $0.42 * * *$ & $0.35^{* * * *}$ & $0.40 * * *$ & $0.29 * *$ \\
\hline GRS F-stat & $4.39 * * *$ & $3.71 * * *$ & $3.25 * *$ & $3.58 * * *$ & $2.61 * *$ \\
\hline ( $p$ value) & $(0.000)$ & $(0.0003)$ & $(0.0016)$ & $(0.0005)$ & $(0.0145)$ \\
\hline
\end{tabular}

The sample consists of all US common stocks (subject to some restrictions as described in the main text) listed on the NYSE, Amex, and NASDAQ from the CRSP-COMPUSTAT merged database. The sample period is between January 1971 and December 2017. We describe the sample in the legend of Table 1. Alpha is the pricing error from various time-series models as described in the main text. The set five factors include excess market return, a size factor, a value factor, an investment factor, and a profitability factor. LMHA and HMLV are as described in the main text. GRS F-stat reports the Gibbons et al. (1989) test statistics and the associated $p$ values. $* * *$, and $* * *$ refer to significance at $10 \%, 5 \%$, and $1 \%$ level respectively

difference between the equal-weighted average of the $\mathrm{L} / \mathrm{L}$ and $\mathrm{L} / \mathrm{H}$ portfolio returns and the equal-weighted average of the $\mathrm{H} / \mathrm{L}$ and $\mathrm{H} / \mathrm{H}$ portfolio returns.

The risk-adjusted abnormal returns for TA-baseddecile portfolios are reported in Table 5. This table also includes the Gibbons, Ross, and Shanken (Gibbons et al. 1989 1989) $\mathrm{F}$ test statistics and the associated $p$ values. ${ }^{17}$ The first set of abnormal returns, reported in column (2) and (3), are the intercepts from models that exclude LMHA and HMLV factors. The alphas related to the $1 \mathrm{~F}$ model suggest that market risk alone is not successful in explaining the abnormal returns of TA-sorted portfolios. The alphas corresponding to the $5 \mathrm{~F}$ model, reported in column (3), suggest that the accrual premium persists after controlling for FF risk factors. The spread in alpha estimates between the

17 The GRS F-statistic is calculated as

$$
\frac{T-N-K}{N}\left[1+E_{T}(f)^{\prime} \widehat{\Omega}^{-1} E_{T}(f)\right]^{-1} \hat{\alpha}^{\prime} \widehat{\Sigma}^{-1} \widehat{\alpha} \sim F_{N, T-N-K}
$$

where $T=$ the length of the time-series, $N=$ number of test portfolios, $K=$ number of factors, $\Omega$ is an unbiased estimate of the factors' covariance matrix, $\widehat{\alpha}$ is a $N \times 1$ vector of estimated intercepts, and $\widehat{\Sigma}$ is an unbiased estimate of the residual covariance matrix. 
low-accrual and high-accrual deciles becomes $0.48 \%$ per month for the $1 \mathrm{~F}$ and $0.42 \%$ per month for the 5F model.

It is not utterly surprising that the abnormal returns of the accrual-sorted portfolios pose special problems. It is known in the literature that for anomalies other than accruals, the $5 \mathrm{~F}$ model improves on the description of average returns provided by the $3 \mathrm{~F}$ model (Fama and French 2016). For accrual-based portfolios, on the other hand, the role of the 5F model is rather limited. One of the key issues is that in the sorts on accruals, while portfolios in the smallest decile have negative slopes for risk factors, they do not exhibit the predicted low average returns. Thus, one-dimensional sorts based on accruals may produce average returns that escape explanation by the risk factor model.

The second set of abnormal returns, reported in column (4) through (6), are the intercepts from the $5 \mathrm{~F}$ model that include either LMHA or HMLV or both. These results suggest that the inclusion of TA- and VaR-based factor-mimicking portfolios result in marginal changes in alpha estimates. In the presence of LMHA, the average alpha becomes smaller, but five out of ten estimates become significant at the 5\% level. When we include HMLV, six out of ten alphas remain statistically different from zero at the 5\% level. When we include both LMHA and HMLV, only four extreme TA portfolios display alphas that are significant at the $5 \%$ level.

In order to test whether all the intercepts are simultaneously zero, we also employ the GRS test for various factor models. We observe that the $1 \mathrm{~F}$ and $5 \mathrm{~F}$ models generate significant GRS statistics of $4.39(p$ value $=0.0000)$ and $3.71(p$ value $=0.0003)$, respectively. Thus, both models reject the null hypothesis that all ten alphas are jointly equal to zero. In comparison, the GRS statistic for three augmented 5F models, reported in the last three columns, are 3.25 ( $p$ value $=0.0016), 3.58(p$ value $=0.0005)$, and 2.61 $(p$ value $=0.0145)$, respectively. The GRS statistic decreases in a multifactor framework that includes either LMHA or HMLV. A 7-factor (7F) model, constructed by augmenting the 5F model with LMHA and HMLV, does better in describing the alpha spread parsimoniously.

In Table 6, we examine the joint significance of alpha estimates for our double- and triple-sorted portfolios. We report the mean absolute and squared intercepts, the GRS statistics and the associated $p$ values. The first two GRS statistics suggest that both $1 \mathrm{~F}$ and $5 \mathrm{~F}$ models miss the abnormal returns of nine TA- and VaR-sorted portfolios. The next three F-statistic suggests that the incorporation of TA- and VaR-based factors does a better job in describing the abnormal returns. For the triple-sorted portfolios, both 5F+LMHA and $5 \mathrm{~F}+\mathrm{HMLV}$ models provide very similar performance metrics. Overall, the 7F model produces the lowest mean absolute and squared alpha, and a GRS statistic that supports the hypothesis that the alphas are jointly insignificant at the $1 \%$ level.

Therefore, our preliminary results suggest that even though the FF common risk factors cannot explain the time-series variability of the accrual premium, the accrual- and VaRbased factor-mimicking portfolios have some limited success. The LMHA and HMLV factors, at a minimum, may play a supplementary role in improving the risk characterization of accrual-based testing assets. In other words, there might be some types of risk captured by individual assets' tail risk, which is independent of conventional systematic risk measures, and is associated with portfolios with extreme accruals. The incorporation of such a proxy of maximum likely loss can therefore aid in the evaluation and interpretation of the accrual anomaly. Firms in extreme accrual portfolios are associated with high VaR, causing the accrual premium to be concentrated in high-risk portfolios, and thus, for riskaverse arbitrageurs, a long-short position on the high- and low-accrual portfolios becomes relatively risky. Given the existing risk-growth interpretations of the accrual anomaly in the 
literature (see e.g., Guo and Jiang 2011; Wu et al. 2009; Zhang 2007), the interpretation of our findings is not outside the domain of financial theory.

\section{Cross-sectional analysis}

Tables 3 and 4 suggest that sorting stocks on variables other than TA, such as VaR, influences the cross-sectional dispersion of average returns. Accordingly, it is imperative to examine whether VaR-based risk factor loading and VaR characteristic are statistically significant in the cross-section after controlling for other determinants of expected stock returns. In this section, we conduct such experiments. First, in Sect. 5.1, we evaluate the determinants of the expected returns of individual stocks using Fama and MacBeth (1973) cross-sectional regressions (CSR). We employ a number of controls of expected returns. These controls include well-known risk factor loadings as well as firm characteristics. The following two subsections examine how much of the accrual premium, observed in the CSR of Sect. 5.1, can be attributable to VaR, and how much of the premium remains unexplained.

\subsection{Fama-MacBeth cross-sectional regressions}

The traditional implementation of Fama-MacBeth two-pass CSR methodology involves two steps. In the first step, the risk loadings of various factors are estimated using timeseries regressions. In the second step, the risk premiums are estimated in CSR across all assets. The estimated betas from step 1 are the exposures to the corresponding factors, and their slopes in step 2 capture the reward for bearing the associated factor risk.

In our case, we follow Fama and French (2008) and Hirshleifer et al. (2012) and regress monthly individual stock returns on factor loadings and a number of cross-sectional characteristics that have been shown to have predictive power for future stock returns. Consequently, our cross-sectional tests provide a basis for evaluating rational factor pricing explanations of the accrual anomaly against the alternative hypothesis of market mispricing. Although the individual stock-level analysis is robust to data mining, concerns about measurement error remain. As has been demonstrated in the literature, the factor loadings of individual stocks in step 1 can be noisy and that results in errors-in-variables problems in Stage 2. To mitigate such errors-in-variables problems, we follow Hou and Moskowitz (2005) and assign the loadings of our size-TA-VaR-sorted portfolios to each individual stock in its respective portfolio. Table 7 reports the time-series averages of the monthly cross-sectional regression coefficients (multiplied by 100) and their t-statistics.

All five models in Panel A implement univariate CSR to determine the influence of accrual- and VaR-based factor loadings (model 1 and 2), and characteristics (model 3 through 5). Model 1 and 2 show that both LMHA and HMLV factor loadings, given by $\beta_{L M H A}$ and $\beta_{H M L V}$ respectively, are positively related to the average returns of individual stocks (the slope of $\beta_{L M H A}$ is 0.194 with t-stat $=2.77$, and the slope of $\beta_{H M L V}$ is 0.168 with $\mathrm{t}$-stat $=2.60$ ). Thus, if LMHA and HMLV serve as proxies for sensitivity to risk fundamentals, they are compensated with higher expected returns. However, this evidence per se does not mean that the market risk explanation holds, because without the incorporation 
Table 6 Summary of intercepts and Gibbons et al. (1989) F-test statistic for various multidimensional portfolios

\begin{tabular}{|c|c|c|c|c|c|}
\hline Dependent portfolios & Explanatory portfolios & $\operatorname{Avg}|\alpha|$ & $\operatorname{Avg} \alpha^{2}$ & $\begin{array}{l}\text { GRS } \\
\text { F-Stat }\end{array}$ & $\begin{array}{l}p \text { value } \\
\text { (GRS) }\end{array}$ \\
\hline \multirow[t]{5}{*}{9 TA-VaR } & 1-Factor & 0.183 & 0.046 & $4.25 * * *$ & 0.0000 \\
\hline & 5-Factor & 0.138 & 0.033 & $3.38 * * *$ & 0.0016 \\
\hline & 5-Factor + LMHA & 0.126 & 0.029 & $2.82 * *$ & 0.0097 \\
\hline & 5-Factor + HMLV & 0.133 & 0.031 & $2.91 * *$ & 0.0073 \\
\hline & 5-Factor + LMHA + HMLV & 0.104 & 0.026 & $2.50 * *$ & 0.0259 \\
\hline \multirow[t]{5}{*}{27 Size-TA-VaR } & 1-Factor & 0.214 & 0.053 & $3.77 * * *$ & 0.0000 \\
\hline & 5-Factor & 0.191 & 0.042 & $3.02 * * *$ & 0.0011 \\
\hline & 5-Factor + LMHA & 0.182 & 0.037 & $2.53 * * *$ & 0.0049 \\
\hline & 5-Factor + HMLV & 0.170 & 0.039 & $2.74 * * *$ & 0.0053 \\
\hline & 5-Factor + LMHA + HMLV & 0.142 & 0.030 & $2.26 * *$ & 0.0167 \\
\hline
\end{tabular}

The sample consists of all US common stocks (subject to some restrictions as described in the main text) listed on the NYSE, Amex, and NASDAQ from the CRSP-COMPUSTAT merged database. The test portfolios consist of a set of nine portfolios based on total accruals and semiparametric VaR, and a set of twentyseven portfolios based on firm size, total accruals and semiparametric VaR. We describe the samples in the legend of Tables 3 and 4. Alpha is the pricing error from various time-series models as described in the main text. The set five factors include excess market return, a size factor, a value factor, an investment factor, and a profitability factor. LMHA and HMLV are long-short portfolios that mimic the risk factor in average returns related to total accruals and VaR, respectively. GRS F-stat reports the Gibbons et al. (1989) test statistics and the associated $p$ values. $* * *$, and $* * *$ refer to significance at $10 \%, 5 \%$, and $1 \%$ level respectively

of accrual and VaR characteristics, one cannot draw a clear conclusion regarding the risk versus mispricing explanations.

In the next three regressions, we control for firm-level characteristics in isolation. In model 3, when we control solely for accrual, that characteristic becomes a strong negative predictor of the average returns of individual stocks (slope $=-1.305$ and t-stat $=-5.84$ ). For tail-risk characteristic, analogous to the use of $\mathrm{ME}$ and $\mathrm{BE} / \mathrm{ME}$ in the literature, we construct log specification of VaR in order to incorporate them into CSR. ${ }^{18}$ In the next two models, we control solely for $\ln (\mathrm{VAR})$. In model (4), the average slope of $\ln \left(\mathrm{VAR}_{5 \%}\right)$ is 0.237 , with a t-statistic of 3.61 , whereas in model (5), the average slope of $\ln \left(\mathrm{VAR}_{1 \%}\right)$ is 0.12 , with a t-statistic of 2.37 . The last two models suggest that $5 \% \mathrm{VaR}$ and $1 \% \mathrm{VaR}$ each have a significant positive influence on average returns. The $\mathrm{R}^{2}$ values in Panel A are small and range from 0.0013 to 0.0059 with accruals generating the highest value.

In Panel B, we implement various multivariate CSR and evaluate the marginal role of each firm-level characteristic. In addition to the reported covariates, all models now include the loadings of FF 5 factors. ${ }^{19}$ The first model includes both LMHA and HMLV factor loadings. It suggests that while $\beta_{L M H A}$ is individually significant at the $5 \%$ level

\footnotetext{
18 We also experiment with other nonlinear specifications of VaR but the results show no substantial improvement over the ones reported in this paper.

19 Following Khan (2008), we include cash-flow news and discount rate news in our cross-sectional tests. We find that, similar to other recent papers, the additional news factors do not explain the accrual premium in our sample.
} 


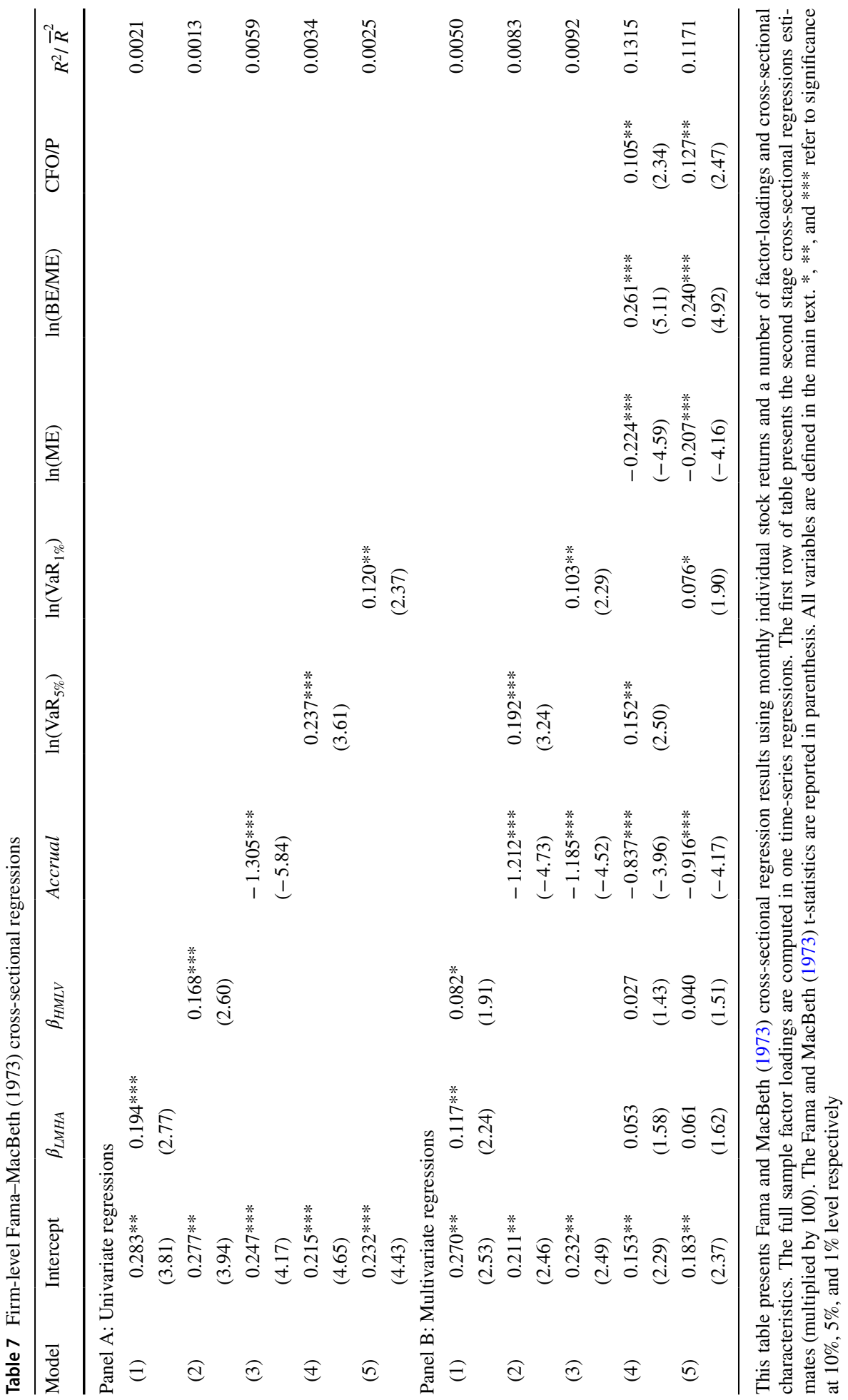


(slope $=0.117$, t-stat $=2.24)$ and $\beta_{H M L V}$ is not $($ slope $=0.082$, t-stat $=1.91)$, both $\beta_{L M H A}$ and $\beta_{H M L V}$ are jointly significant in the cross-section $(\mathrm{F}-\mathrm{stat}=7.03, p$ value $=0.000)$. The next two models incorporate accrual and VaR characteristics simultaneously. Model (2) suggests that the coefficient of the accrual remains a highly significant predictor of average returns and that $\ln \left(\mathrm{VAR}_{5 \%}\right)$ neither subsumes nor is subsumed by the accrual. A similar result also holds for $\ln \left(\mathrm{VAR}_{1 \%}\right)$ in model (3), although the effect of $\ln \left(\mathrm{VAR}_{1 \%}\right)$ is not as strong when accrual is included. While $\ln \left(\mathrm{VAR}_{5 \%}\right)$ remains statistically significant at the $1 \%$ level in the presence of accruals, $\ln \left(\mathrm{VAR}_{1 \%}\right)$ becomes significant only at the $5 \%$ level. $^{20}$

The last two models of Panel B include the loadings of LMHA and HMLV in the presence of $\ln (\mathrm{ME}), \ln (\mathrm{BE} / \mathrm{ME}), \mathrm{CFO} / \mathrm{P}$, accrual, and VaR. Model (4) shows that all firmlevel characteristics remain a highly significant predictor of average returns, whereas the loading of the long-short portfolios diminish and are not statistically different from zero. Both $\beta_{L M H A}$ and $\beta_{H M L V}$ are no longer jointly priced in the cross-section (F-stat $=3.17, p$ value $=0.016$ ). In model (5), all of our previous results hold, with the exception of $\ln \left(\mathrm{VAR}_{1 \%}\right)$ only being significant at the $10 \%$ level. Model (5) also generates the highest $\bar{R}^{2}$. Statistically, accrual remains the most precise predictor, and 5\% VaR always demonstrates a stronger association than $1 \% \mathrm{VaR}$. All algebraic signs of the slope coefficients associated with factor loadings and characteristics seem plausible and consistent with their prior expectations.

It is worth pointing out that our objective in the paper is not to evaluate the effect of financial crisis on VaR estimate, rather we are interested in examining the significance of accrual effect in the presence of VaR. One can always argue whether the estimation of VaR using time-series model (2) is sensitive to the sample size or, the historical data used. For example, a sample period covering a financial crisis would produce different GARCH parameters and hence different VaR. If one wants to avoid the use of financial crisis in the VaR calculation, then that necessitates the exclusion of firm characteristics such as size, book-to-market, and accruals from the same sample period. All these can cause serious data snooping bias and raise various methodological issues. It can also result in misleading empirical tests about whether the accrual effect is a result of market mispricing or of risk fundamentals. ${ }^{21}$

Altogether, our firm-level CSR results demonstrate that the TA- and VaR-based factor loadings are compensated with higher expected returns only in the absence of firm characteristics. When we control for firm-level accrual and VaR, the loading of LMHA and HMLV do not predict average stock returns. Consistent with our previous findings, firmlevel VaR is positively related to average returns, while accrual is inversely related. In sum, our CSR results are consistent with the market mispricing explanation and not with the

\footnotetext{
${ }^{20}$ In unreported results, we also include the interaction term Accruals $\times \ln (\mathrm{VaR})$ in the CSR of Panel B. If the slope of the interaction is positive, the accrual and VaR effects would be substitutes. On the other hand, if the slope is negative, these two effects would be complements. We find that the estimated coefficient on the interactive term is negative and statistically significant only at the $10 \%$ level, suggesting that the two effects are weakly independent.

21 Moreover, even though some financial crises may look extreme, statistically they are not quite unusual or abnormal. For example, during the global financial crisis of 2007-08, the annual return of the US stock market was $-39.95 \%$ for the year 2008 . If we assume that the annual stock market returns follow a normal distribution, then the probability of a return of $-39.95 \%$ or smaller is around $1.20 \%$. In addition, using the estimated mean and the standard deviation for the 1927-2008 period, the annual return $-39.95 \%$ is only -2.26 standard deviations away from the mean. Thus, statistically the large loss for the year 2008 is uncommon, but not out of bounds.
} 
risk-based rational factor pricing explanation. The main inference of this paper that the accrual anomaly is driven by mispricing is supported by the existing work of Hirshleifer et al. (2012) and Ohlson and Bilinski (2015).

\subsection{Modified Hou and Loh (2016) decomposition methodology: univariate analysis}

The CSR results from the previous subsection highlights the importance of firm-level accrual and tail risk, but they are not informative enough to indicate how much of the accrual premium is explained by tail risk. In this subsection, we follow Hou and Loh (2016) and decompose the accrual premium into two components: one related to VaR and the other a residual component. The decomposition methodology supports our conclusion that the accrual anomaly derives from market mispricing and not from risk fundamentals. Briefly, following Hou and Loh (2016), we design a methodology consisting of three stages.

Stage 1: We estimate FM CSR of month t excess returns of individual stocks on each firm's accrual of month $t-1$ by

$$
R_{i, t}=\alpha_{t}+b_{t} \text { Accrual }_{i, t-1}+\varepsilon_{i, t}
$$

Stage 2: For each firm, we regress the accrual in month $t-1$ on the VaR measure in month $\mathrm{t}-1$ using

$$
\operatorname{Accrual}_{i, t-1}=a_{t-1}+\delta_{t-1} \ln \left(\operatorname{VaR}_{i, t-1}\right)+e_{i, t-1}
$$

Stage 3: We decompose the accrual premium $b_{t}$, obtained from stage 1 , into two orthogonal components

$$
\begin{aligned}
b_{t} & =\frac{\operatorname{Cov}\left(R_{i, t}, \text { Accrual }_{i, t-1}\right)}{\operatorname{Var}\left(\text { Accrual }_{i, t-1}\right)}=\frac{\operatorname{Cov}\left(R_{i, t}, a_{t-1}+\delta_{t-1} \ln \left(\operatorname{VaR}_{i, t-1}\right)+e_{i, t-1}\right)}{\operatorname{Var}\left(\operatorname{Accrual}_{i, t}\right)} \\
& =\frac{\operatorname{Cov}\left(R_{i, t}, \delta_{t-1} \ln \left(\operatorname{VaR}_{i, t-1}\right)\right)}{\operatorname{Var}\left(\text { Accrual }_{i, t-1}\right)}+\frac{\operatorname{Cov}\left(R_{i, t}, a_{t-1}+e_{i, t-1}\right)}{\operatorname{Var}\left(\text { Accrual }_{i, t-1}\right)} \\
& =b_{t}^{1}+b_{t}^{2}
\end{aligned}
$$

where $\frac{b_{t}^{1}}{b_{t}}$ is the fraction of the accrual premium explained by $\ln \left(\operatorname{VaR} R_{i, t-1}\right)$ and $\frac{b_{t}^{2}}{b_{t}}$ is the fraction that remains unexplained by $\mathrm{VaR}$.

In Table 8, we examine the results of our decomposition to assess what fraction of the accrual premium is captured by the VaR and by the residual component. In addition to our full sample of the NYSE-AMEX-NASDAQ universe, we also replicate the results using stocks with NYSE-AMEX listings. Following Hou and Loh (2016), the risk premiums are calculated using the time-series averages of the monthly cross-sectional regressions of the monthly excess stock returns on each firm's accruals. Each row reports the decomposition results of the stage 1 accruals slope coefficient related to $\ln \left(\mathrm{VAR}_{5 \%}\right)$ (or $\ln \left(\mathrm{VAR}_{1 \%}\right)$ ) and the residual component.

The results corresponding to $\ln \left(\mathrm{VAR}_{5 \%}\right)$ are reported first, followed by $\ln \left(\mathrm{VAR}_{1 \%}\right)$. The time-series average estimates of $b_{t}^{1}$ and $b_{t}^{2}$ are $-0.187(\mathrm{t}-\mathrm{stat}=-6.58)$ and -1.091 $(\mathrm{t}$-stat $=-23.04)$, respectively. Thus, the fraction of the accrual coefficient from stage 1 that is attributable to $\ln \left(\mathrm{VAR}_{5 \%}\right)$ is around $14.39 \%(\mathrm{t}=7.73)$, and the fraction attributable to the residual component is $85.61 \%(\mathrm{t}=64)$. For $\ln \left(\mathrm{VAR}_{1 \%}\right)$, the time-series average estimates of 


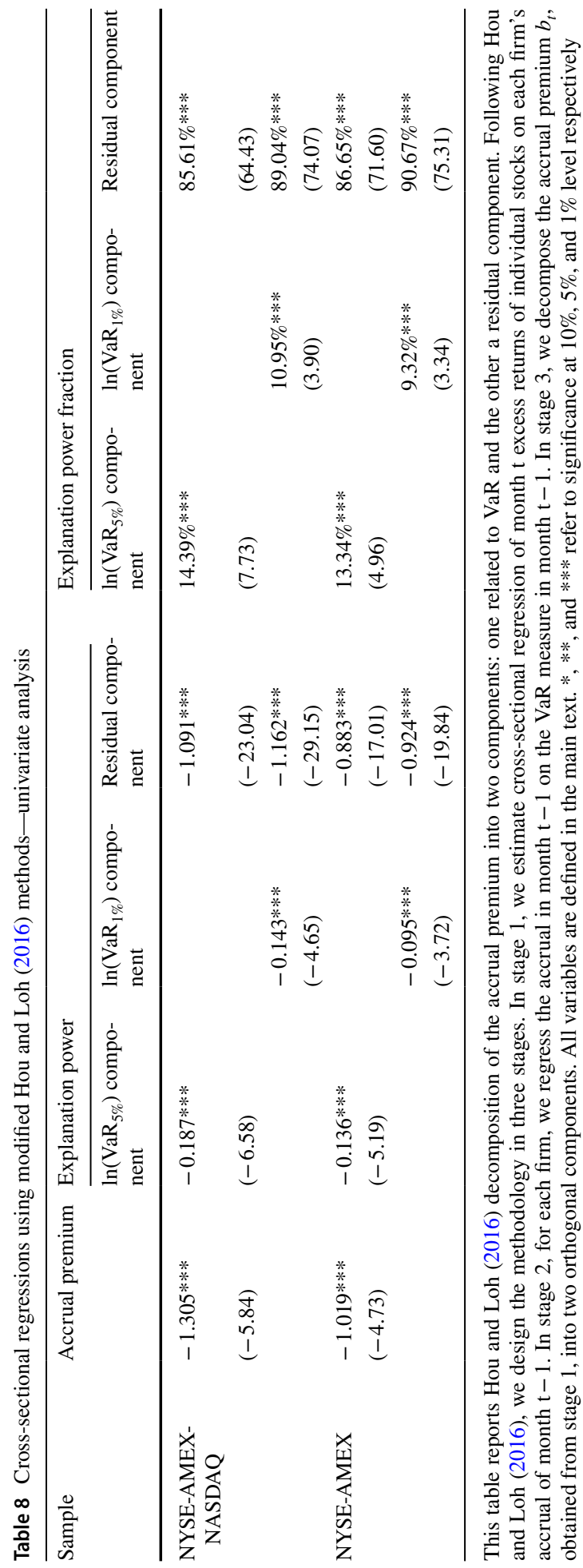


$b_{t}^{1}$ and $b_{t}^{2}$ are $-0.143(\mathrm{t}-\mathrm{stat}=-5.19)$ and $-1.162(\mathrm{t}-\mathrm{stat}=-29.15)$, respectively. Although, $\ln \left(\mathrm{VAR}_{1 \%}\right)$ does not explain the average stock returns well (recall $\mathrm{R}^{2}=0.0025$ in model (5) of Table 7 Panel A), around 10\% of the accrual premium can be attributable to this tail-risk component. For the NYSE-AMEX stocks, the results show no substantial changes and the accrual premium becomes slightly smaller $(-1.019)$ but remain highly significant $(\mathrm{t}=-4.73)$. Taken together, $\ln \left(\mathrm{VAR}_{5 \%}\right)$ explains around $13 \%$ while $\ln \left(\mathrm{VAR}_{1 \%}\right)$ explains around $9 \%$ of the accrual premium for stocks with non-NASDAQ listings. Thus, the individual firms' VaR explains the accrual premium with limited success.

\subsection{Multivariate decomposition analysis}

In the previous subsection, we investigated the role of $\mathrm{VaR}$ in isolation. Now we examine the marginal contribution of $\mathrm{VaR}$ in the presence of other competing variables and conduct a multivariate decomposition analysis under the Hou and Loh (2016) framework. We include four candidate variables $\ln (\mathrm{ME}), \ln (\mathrm{BE} / \mathrm{ME}), \mathrm{CFO} / \mathrm{P}$, and $\ln (\mathrm{VaR})$ simultaneously ${ }^{22}$ and design the methodology in three stages. In stage 1, we estimate the cross-sectional regression of month t excess returns of individual stocks on each firm's accrual of month $\mathrm{t}-1$. In stage 2 , for each firm, we regress the accrual in month $\mathrm{t}-1$ on four characteristics in month $t-1$. In stage 3 , we decompose the accrual premium, obtained from stage 1 , into five orthogonal components. Table 9 report the results.

For the full sample, the set of four characteristics that include $\ln \left(\mathrm{VAR}_{5 \%}\right)$ explain around $53 \%$ of the accrual premium, and the residual component accounts for the rest. In the presence of three additional characteristics, $\ln \left(\mathrm{VAR}_{5 \%}\right)$ captures around $9.53 \%$ of the accrual premium, while $\ln \left(\mathrm{VAR}_{1 \%}\right)$ explains $8.13 \%$. Thus, the marginal role of $\mathrm{VaR}$ is smaller in a multivariate framework. In terms of the marginal role of individual characteristics, $\ln (\mathrm{BE} /$ ME) contributes the largest-as it always captures at least $23 \%$ of the accrual premium. In contrast, $\ln (\mathrm{ME})$ is the second largest contributor. Even in the presence of $\ln (\mathrm{BE} / \mathrm{ME})$, $\mathrm{CFO} / \mathrm{P}$ and $\ln \left(\mathrm{VAR}_{5 \%}\right)$, the fraction of the accrual premium explained by $\ln (\mathrm{ME})$ is $18.26 \%$ $(t=3.89)$ for the full sample and $15.09 \%(t=3.57)$ for the non-NASDAQ sample. Regardless of the constituents of the sample, the CFO/P contribution is smallest. When $\ln \left(\mathrm{VAR}_{5 \%}\right)$ and others are included, the $\mathrm{CFO} / \mathrm{P}$ captures $5.73 \%(\mathrm{t}=1.90)$ for the full sample and $3.70 \%$ $(t=1.75)$ for the NYSE-AMEX accrual premium. For both $\ln \left(\mathrm{VAR}_{5 \%}\right)$ and $\ln \left(\mathrm{VAR}_{1 \%}\right)$, the explained fraction from multivariate analysis is lower than its univariate counterpart, although they remain statistically significant at the $5 \%$ level.

In short, the above decompositions show that our firm-level VaR, along with other recognized characteristics, can be a relevant contributor to the accrual anomaly. The effect of $\mathrm{VaR}$ on the accrual premium is small but survives the presence of cross-sectional characteristics that are known to be related to accruals and documented previously in the literature. Similar decomposition methodology can be used in the context of other asset pricing anomalies.

${ }^{22}$ The analysis can be extended to include other possible explanatory variables. 


\section{Conclusions}

In the literature, statistically significant risk-adjusted abnormal returns of accrual-sorted portfolios pose exceptional challenges to rational factor pricing. In this paper, we examine the relative importance of individual-assets' tail risk-as opposed to aggregate tail risk or systematic risk measures that are common to many assets-in the return dynamics of accrual-sorted portfolios. We utilize dynamic time-series models that jointly parameterize conditional means and conditional variances and estimate the VaR of an asset (or a portfolio of assets) over a given time period for a given confidence level. We investigate whether individual assets' maximum likely loss measure, proxied by VaR, captures the mispricing attributes of the accrual anomaly. We construct portfolios using firm-level accrual and VaR to provide insights about the possible contributing role of $\mathrm{VaR}$ on the accrual effect. Consistent with our intuition, VAR is positively related to average returns in the cross-section, while firm-level accrual is inversely related. The negative association between accruals and average returns decreases once we control for VaR.

Our univariate cross-sectional regression results demonstrate that the accrual- and VaRbased factor loadings are compensated with higher expected returns only in the absence of firm characteristics. In our multivariate cross-sectional regression analysis, firm-level accrual and VaR remain a highly significant predictor of average returns, and VaR neither subsumes nor is subsumed by accrual. Similar to Hou and Loh (2016), we also conduct a cross-sectional decomposition analysis and it suggests that, for all CRSP stocks, the 5\% VaR component explains about $14 \%$ of the accrual premium, while the $1 \%$ VaR component, in contrast, captures around $11 \%$. To examine whether VaR maintains its role in other sample and in the presence of competing characteristics, we also utilize stocks from nonNASDAQ listings and perform a multivariate decomposition analysis. Taken together, our estimates show that firm-level VaR continues to capture at least $7 \%$ of the accrual premium even in the presence of size and $\mathrm{BE} / \mathrm{ME}$ characteristics. The fact that the maximum likely loss provides a support for the mispricing explanation of the accrual anomaly is not recognized in the literature.

\section{Appendix: Details of value-at-risk (VaR)}

Let $R_{i, t}$ is the return of asset $i$ in excess of the risk-free rate at time $t$. For a given timeseries of returns on the asset $\left\{R_{i, t}\right\}_{t=1}^{n}$, the $100 \tau \%$ VaR at time $\mathrm{t}$ is defined by

$$
\operatorname{Prob}\left(R_{i, t}<-\operatorname{VaR} \mid \mathcal{F}_{t-1}\right)=\tau
$$

where $\mathcal{F}_{t-1}$ denotes the information set at time $t-1$, which may include past values of $R_{i, t}$ and possibly some covariates. Typically, the confidence level is exceeded with probability $1-\tau$, where $\tau$ is often set at $0.01,0.05$, or 0.10 .

There are several VaR estimation methods in the literature. The most common approach is the non-parametric method, where no assumptions are made regarding the distribution of returns and an estimate of $\mathrm{VaR}$ is calculated based on the lower tail of the actual empirical distribution. Another approach is the parametric VaR, where some assumptions are made regarding the specific distribution of returns. For example, if we assume that the return distribution is conditionally normal, the $1 \% \mathrm{VaR}$ at time $\mathrm{t}$ can be computed as 
Table 9 Cross-sectional regressions using modified Hou and Loh (2016) methods-multivariate analysis

\begin{tabular}{|c|c|c|c|c|c|c|c|c|}
\hline & \multicolumn{4}{|c|}{ Explanation power } & \multicolumn{4}{|c|}{ Explanatory power fraction } \\
\hline & \multicolumn{2}{|c|}{ NYSE-AMEX-NASDAQ } & \multicolumn{2}{|c|}{ NYSE-AMEX } & \multicolumn{2}{|c|}{$\begin{array}{l}\text { NYSE-AMEX-NAS- } \\
\text { DAQ }\end{array}$} & \multicolumn{2}{|c|}{ NYSE-AMEX } \\
\hline $\ln (\mathrm{ME})$ & $\begin{array}{l}-0.238^{* * *} \\
(-3.13)\end{array}$ & $\begin{array}{l}-0.201 * * * \\
(-2.88)\end{array}$ & $\begin{array}{l}-0.197 * * * \\
(-2.90)\end{array}$ & $\begin{array}{l}-0.175^{* * * *} \\
(-2.77)\end{array}$ & $\begin{array}{l}18.26^{* * * *} \\
(3.89)\end{array}$ & $\begin{array}{l}19.71 * * * \\
(4.42)\end{array}$ & $\begin{array}{l}15.09^{* * * *} \\
(3.57)\end{array}$ & $\begin{array}{l}17.19 * * * \\
(4.06)\end{array}$ \\
\hline $\ln (\mathrm{BE} / \mathrm{ME})$ & $\begin{array}{l}-0.297 * * * \\
(-3.92)\end{array}$ & $\begin{array}{l}-0.250 * * * \\
(-3.27)\end{array}$ & $\begin{array}{l}-0.306 * * * \\
(-3.85)\end{array}$ & $\begin{array}{l}-0.265 * * * \\
(-3.59)\end{array}$ & $\begin{array}{l}22.74 * * * \\
(5.09)\end{array}$ & $\begin{array}{l}24.56 * * * \\
(5.72)\end{array}$ & $\begin{array}{l}23.41 * * * \\
(5.81)\end{array}$ & $\begin{array}{l}26.03 * * * \\
(6.49)\end{array}$ \\
\hline $\mathrm{CFO} / \mathrm{P}$ & $\begin{array}{l}-0.075^{* *} \\
(-2.15)\end{array}$ & $\begin{array}{l}-0.062 * * \\
(-2.03)\end{array}$ & $\begin{array}{l}-0.049 * \\
(-1.87)\end{array}$ & $\begin{array}{l}-0.048^{*} \\
(-1.82)\end{array}$ & $\begin{array}{l}5.73^{*} \\
(1.90)\end{array}$ & $\begin{array}{l}6.08 * * \\
(1.98)\end{array}$ & $\begin{array}{l}3.70^{*} \\
(1.75)\end{array}$ & $\begin{array}{l}4.68 * \\
(1.83)\end{array}$ \\
\hline $\ln \left(\mathrm{VaR}_{5 \%}\right)$ & $\begin{array}{l}-0.124 * * \\
(-2.49)\end{array}$ & & $\begin{array}{l}-0.146 * * \\
(-2.55)\end{array}$ & & $\begin{array}{l}9.53 * * \\
(2.26)\end{array}$ & & $\begin{array}{l}11.25^{* *} \\
(2.39)\end{array}$ & \\
\hline $\ln \left(\mathrm{VaR}_{1 \%}\right)$ & & $\begin{array}{l}-0.083 * * \\
(-2.35)\end{array}$ & & $\begin{array}{l}-0.075 * * \\
(-2.18)\end{array}$ & & $\begin{array}{l}8.13 * * \\
(2.19)\end{array}$ & & $\begin{array}{l}7.36 * * \\
(2.07)\end{array}$ \\
\hline Residual & $\begin{array}{l}-0.611^{* * *} \\
(-4.82)\end{array}$ & $\begin{array}{l}-0.440 * * * \\
(-4.06)\end{array}$ & $\begin{array}{l}-0.633 * * * \\
(-4.73)\end{array}$ & $\begin{array}{l}-0.469 * * * \\
(-4.15)\end{array}$ & $\begin{array}{l}46.81^{* * * *} \\
(9.64)\end{array}$ & $\begin{array}{l}43.22 * * * \\
(8.30)\end{array}$ & $\begin{array}{l}48.54 * \\
(11.05)\end{array}$ & $\begin{array}{l}46.05 * * * \\
(10.37)\end{array}$ \\
\hline
\end{tabular}

The table reports Hou and Loh (2016) multivariate decomposition of the accrual premium into five components: four related to firm-level characteristics and the other a residual component. Following Hou and Loh (2016), we design the methodology in three stages. In stage 1, we estimate the cross-sectional regression of month $t$ excess returns of individual stocks on each firm's accrual of month $t-1$. In stage 2 , for each firm, we regress the accrual in month $t-1$ on four characteristics in month $t-1$. In stage 3 , we decompose the accrual premium, obtained from stage 1, into five orthogonal components. All variables are defined in the main text. *,**, and $* * *$ refer to significance at $10 \%, 5 \%$, and $1 \%$ level respectively

$$
-\left(\mu_{i, t}-2.576 * \sqrt{h_{i, t}}\right)
$$

where $\mu_{i, t}$ and $\sqrt{h_{i, t}}$ are the conditional mean and conditional volatility of $R_{i, t}$, and 2.576 is the critical value derived from the standard normal distribution corresponding to the 99\% confidence level. Finally, there exists an alternative approach based on the semi-parametric method, which makes assumptions about the model dynamics but not the return distribution.

One of the major challenges in any type of empirical implementation of VaR is the precise measurement of $\mu_{i, t}$ and $h_{i, t}$. Approaches such as the one used by RiskMetrics take a simple weighted average to model $h_{i, t}$. For example, in RiskMetrics, the forecast for time $\mathrm{t}$ variance is given by $h_{i, t}=\lambda h_{i, t-1}+(1-\lambda) r_{i, t-1}^{2}$, where the parameter $\lambda$ is the decay factor, which RiskMetrics sets at 0.94 for daily data and 0.97 for monthly data. To capture the time-varying estimate of $\mu_{i, t}$ and $h_{i, t}$, a viable alternative is to explore a range of parametric models in addition to specific assumptions about return distribution. For example, if we assume that the time-series of a return is modeled by

$$
R_{i, t}=\mu_{i, t}+\sqrt{h_{i, t}} \varepsilon_{i, t}
$$

the conditional $\mathrm{VaR}$ of $R_{i, t}$ given $\mathcal{F}_{t-1}$ is

$$
\operatorname{VaR}_{t}(\tau)=\mu_{i, t}+\sqrt{h_{i, t}} Q_{\varepsilon}(\tau)
$$

where $Q_{\varepsilon}(\tau)$ denotes the unconditional VaR of the error term $\varepsilon_{i, t}$. 
In this paper, we use two measures of VaR. The primary measure is semiparametric VaR, which uses QML-GARCH predictions, and the secondary measure is parametric VaR, which uses standard normal-GARCH predictions. For both methods, we jointly parameterize conditional means and conditional variances by the $\operatorname{AR}(1)+\operatorname{GARCH}(1,1)$ specification (2), given in Sect. 3, to describe the time-series of $R_{i, t}$. For the semiparametric method, we follow Engle and Manganelli $(2001,2004)$ and first fit model (2) for each individual asset returns using the QML method and then compute the VaR from Eq. (3), given in Sect. 3, by multiplying the empirical quantile of the standardized residuals by $\sqrt{\widehat{h}_{i, t}}$. For the parametric method, after estimating the parameters of model (2) by maximum likelihood, we obtain the $100 \tau \%$ VaR at time $\mathrm{t}$ by the estimated GARCH variance times the $\tau$-quantile of the standard normal distribution.

\section{References}

Aboura S, Arisoy YE (2019) Can tail risk explain size, book-to-market, momentum, and idiosyncratic volatility anomalies? J Bus Finance Account 46(9-10):1263-1298

Agarwal V, Ruenzi S, Weigert F (2017) Tail risk in hedge funds: a unique view from portfolio holdings. J Financ Econ 125(3):610-636

Allen L, Bali TG, Tang Y (2012) Does systemic risk in the financial sector predict future economic downturns? Rev Financ Studies 25(10):3000-3036

Ali A, Gurun UG (2009) Investor sentiment, accruals anomaly, and accruals management. J Account Audit Financ 24(3):415-431

Almeida C, Ardison K, Garcia R, Vicente J (2017) Nonparametric tail risk, stock returns, and the macroeconomy. J Financ Econ 15(3):333-376

Andersen T, Fusari N, Todorov V (2020) The pricing of tail risk and the equity premium: evidence from international option markets. J Bus Econ Stat 38(3):662-678

Avramov D, Chordia T, Jostova G, Philipov A (2013) Anomalies and financial distress. J Financ Econ 108(1):139-159

Báez-Díaz A, Alam P (2013) Tax conformity of earnings and the pricing of accruals. Rev Quant Finance Account 40(3):509-538

Bali T, Cakici N (2004) Value at risk and expected stock returns. Financ Anal J 60(2):57-73

Bali T, Gokcan S, Liang B (2007) Value at risk and the cross-section of hedge fund return. J Bank Finance 31(4):1135-1166

Bali T, Cakici N, Whitelaw RF (2014) Hybrid tail risk and expected stock returns: when does the tail wag the dog? Rev Asset Pricing Stud 4(2):206-246

Ball R, Gerakos J, Linnainmaa JT, Nikolaev V (2016) Accruals, cash flows, and operating profitability in the cross section of stock returns. J Financ Econ 121(1):28-45

Bams D, Blanchard G, Lehnert T (2017) Volatility measures and value-at-risk. Int J Forecast 33(4):848-863

Barth M, Hutton A (2004) Analyst earnings forecast revisions and the pricing of accruals. Rev Account Stud 9(1):59-96

Bartov E, Kim M (2004) Risk, mispricing, and value investing. Rev Quant Finance Account 23(4):353-376

Bollerslev T, Todorov V (2011) Tails, fears, and risk premia. J Finance 66(6):2165-2211

Bollerslev T, Woolridge JM (1992) Quasi-maximum likelihood estimation and inference in dynamic models with time varying covariances. Econ Rev 11(2):143-172

Bollerslev T, Todorov V, Xu L (2015) Tail risk premia and return predictability. J Financ Econ 118(1):113-134

Bradshaw M, Richardson S, Sloan R (2001) Do analysts and auditors use information in accruals? J Account Res 39(1):45-74

Carhart M (1997) On persistence in mutual fund performance. J Finance 52(1):57-82

Chapman DA, Gallmeye MF, Martin JS (2018) Aggregate tail risk and expected returns. Rev Asset Pricing Stud 8(1):36-76

Daniel KD, Jagannathan R, Kim S (2012) Tail risk in momentum strategy returns. National Bureau of Economic Research (NBER). http://www.nber.org/papers/w18169.pdf 
Dechow P, Dichev I (2002) The quality of accruals and earnings: the role of accrual estimation errors. Account Rev 77(s-1):35-59

Dechow P, Sloan R, Zha Z (2014) Stock prices and earnings: a history of research. Annu Rev Financ Econ 6(1):343-363

Desai H, Rajgopal S, Venkatachalam M (2004) Value-glamour and accruals mispricing: one anomaly or two? Account Rev 79(2):355-385

Detzel A, Schaberi P, Strauss J (2018) There are two very different accrual anomalies. Eur Financ Manag 24(4):581-609

Dopuch N, Seethamraju C, Xu W (2010) The pricing of accruals for profit and loss firms. Rev Quant Finance Account 34(4):505-516

Doukakis LC, Papanastasopoulos GA (2014) The accrual anomaly in the U.K. stock market: implications of growth and accounting distortions. J Int Financ Mark Inst Money 32(1):256-277

Embrechts P, Kluppelberg C, Mikosch T (1997) Modelling external events for insurance and finance. Springer, New York

Engle RF, Manganelli S (2001) Value at risk models in finance. European Central Bank Working Papers, No. 75

Engle RF, Manganelli S (2004) CAViaR: conditional autoregressive value at risk by regression quantiles. J Bus Econ Stat 22(4):367-381

Fama EF, French KR (2008) Dissecting anomalies. J Financ 63(4):1653-1678

Fama EF, French KR (2016) Dissecting anomalies with a five-factor model. Rev Financ Stud 29(1):69-103

Fama EF, MacBeth JD (1973) Risk, return and equilibrium: empirical tests. J Polit Econ 81(3):607-636

Financial Accounting Standards Board (1978) Statement of financial accounting concepts no. 1. Objectives of financial reporting by business enterprises. FASB, Norwalk, CT

Gerlach R, Chen CWS, Chan NYC (2011) Bayesian time-varying quantile forecasting for value-at-risk in financial markets. J Bus Econ Stat 29(4):481-492

Giacomini R, Komunjer I (2005) Evaluation and combination of conditional quantile forecasts. J Bus Econ Stat 23(4):416-431

Gibbons M, Ross S, Shanken J (1989) A test of the efficiency of a given portfolio. Econometrica 57(5):1121-1152

Graham B, Dodd DL (1934) Security analysis, 1st edn. Whittlesey House McGraw-Hill Book Company, New York

Green J, Hand JRM, Soliman MT (2012) Going, going, gone? The demise of the accrual anomaly. Manag Sci 57(5):797-816

Gu M (2020) Distress risk, investor sophistication, and accrual anomaly. J Account Audit Financ 35(1):79-105

Guo H, Jiang X (2011) Accruals and the conditional equity premium. J Account Res 49(1):187-221

Hansen PR, Lunde A (2005) A forecast comparison of volatility models: does anything beat a GARCH(1,1)? J Appl Econ 20(7):873-889

Hirshleifer D, Jiang D (2010) A financing-based misvaluation factor and the cross-section of expected returns. Rev Financ Stud 23(9):3401-3436

Hirshleifer D, Teoh SH, Yu JJ (2011) Short arbitrage, return asymmetry, and the accrual anomaly. Rev Financ Stud 24(7):2429-2461

Hirshleifer D, Hou K, Teoh SH (2012) The accrual anomaly: risk or mispricing? Manag Sci 58(2):320-335

Hou K, Loh R (2016) Have we solved the idiosyncratic volatility puzzle? J Financ Econ 121(1):167-194

Hou K, Moskowitz TJ (2005) Market frictions, price delay, and the cross-section of expected returns. Rev Financ Stud 18(3):981-1020

Hou K, Xue C, Zhang L (2015) Digesting anomalies: an investment approach. Rev Financ Stud 28(3):650-750

Hou K, Xue C, Zhang L (2020) Replicating anomalies. Rev Financ Stud 33(5):2019-2133

Kelly B, Jiang H (2014) Tail risk and asset prices. Rev Financ Stud 27(10):2841-2871

Khan M (2008) Are accruals mispriced? Evidence from tests of an intertemporal capital asset pricing model. J Account Econ 45(1):55-77

Lewellen J, Resutek RJ (2016) The predictive power of investment and accruals. Rev Account Stud 21(4):1046-1080

Mashruwala C, Rajgopal S, Shevlin T (2006) Why is the accrual anomaly not arbitraged away? The role of idiosyncratic risk and transaction costs. J Account Econ 42(1-2):3-33

Neely CJ (2009) Forecasting foreign exchange volatility: why is implied volatility biased and inefficient? And does it matter? J Int Financ Mark Inst Money 19(1):188-205 
Novy-Marx R (2013) The other side of value: the gross profitability premium. J Financ Econ 108(1):1-28 Ohlson JA, Bilinski P (2015) Risk versus anomaly: a new methodology applied to accruals. Account Rev 90(5):2057-2077

Papanastasopoulos GA (2014) Accounting accruals and stock returns: evidence from European equity markets. Eur Account Rev 23(4):729-768

Pastor L, Stambaugh RF (2003) Liquidity risk and expected stock returns. J Polit Econ 111(3):642-685

Pincus M, Rajgopal S, Venkatachalam M (2007) The accrual anomaly: international evidence. Account $\operatorname{Rev} 82(1): 169-203$

Richardson S (2003) Earnings quality and short-sellers. Account Horiz 17(Supplement):49-61

Richardson S, Sloan R, Soliman M, Tuna I (2005) Accrual reliability, earnings persistence, and stock prices. J Account Econ 39(3):437-485

Sloan R (1996) Do stock prices fully reflect information in accruals and cash flows about future earnings? Account Rev 71(3):289-315

Stambaugh RF, Yuan Y (2017) Mispricing factors. Rev Financ Stud 30(4):1270-1315

Stambaugh RF, Yu J, Yuan Y (2012) The short of it: investor sentiment and anomalies. J Financ Econ 104(2):288-302

Teoh S, Wong TJ (2001) Why new issues and high accrual firms underperform? The role of analyst credulity. Rev Financ Stud 15(3):859-900

Tsay RS (2013) An introduction to analysis of financial data with R. Wiley, Hoboken

Van Oordt MRK, Zhou C (2016) Systematic tail risk. J Financ Quant Anal 51(2):685-705

Wu J, Zhang L, Zhang X (2010) The q-theory approach to understanding the accrual anomaly. J Account Res 48(1):177-223

Xiong JX, Idzorek TM, Ibbotson RG (2014) Volatility versus tail risk: which one is compensated in equity funds? J Portf Manag 40(2):112-121

Zhang XF (2007) Accruals, investment, and the accrual anomaly. Account Rev 82(5):1333-1363

Publisher's Note Springer Nature remains neutral with regard to jurisdictional claims in published maps and institutional affiliations. 